\title{
Cluster observations in the magnetosheath - Part 1: Anisotropies of the wave vector distribution of the turbulence at electron scales
}

\author{
A. Mangeney ${ }^{1}$, C. Lacombe $^{1}$, M. Maksimovic ${ }^{1}$, A. A. Samsonov $^{2}$, N. Cornilleau-Wehrlin ${ }^{3}$, C. C. Harvey ${ }^{4}$, \\ J.-M. Bosqued ${ }^{4}$, and P. Trávníček ${ }^{5}$ \\ ${ }^{1}$ LESIA/CNRS, Observatoire de Paris, Meudon, France \\ ${ }^{2}$ Institute of Physics, St. Petersburg State University, St. Petersburg, Russia \\ ${ }^{3}$ Centre d'étude des Environnements Terrestre et Planétaire/UVSQ, Vélizy, France \\ ${ }^{4}$ Centre d'Etude Spatiale des Rayonnements/CNRS, Toulouse, France \\ ${ }^{5}$ Institute of Atmospheric Physics, Prague, Czech Republic
}

Received: 21 April 20-06 - Revised: 23 October 2006 - Accepted: 10 November 2006 - Published: 21 December 2006

\begin{abstract}
We analyse the power spectral density $\delta B^{2}$ and $\delta E^{2}$ of the magnetic and electric fluctuations measured by Cluster 1 (Rumba) in the magnetosheath during $23 \mathrm{~h}$, on four different days. The frequency range of the STAFF Spectral Analyser ( $f=8 \mathrm{~Hz}$ to $4 \mathrm{kHz}$ ) extends from about the lower hybrid frequency, i.e. the electromagnetic (e.m.) range, up to about 10 times the proton plasma frequency, i.e. the

in the magnetosheath result from the Doppler shift of a spatial turbulence frozen in the plasma, and that the intensity of the turbulent $\boldsymbol{k}$ spectrum is strongly anisotropic, for both e.m. and e.s. fluctuations. We conclude that the turbulence has strongly anisotropic $\boldsymbol{k}$ distributions, on scales ranging from $k c / \omega_{p e} \simeq 0.3(50 \mathrm{~km})$ to $k \lambda_{D e} \simeq 1(30 \mathrm{~m})$, i.e. at electron scales, smaller than the Cluster separation.
\end{abstract} electrostatic (e.s.) range. In the e.m. range, we do not consider the whistler waves, which are not always observed, but rather the underlying, more permanent fluctuations. In this e.m. range, $\delta B^{2}$ (at $10 \mathrm{~Hz}$ ) increases strongly while the local angle $\Theta_{B V}$ between the magnetic field $\boldsymbol{B}$ and the flow velocity $\boldsymbol{V}$ increases from $0^{\circ}$ to $90^{\circ}$. This behaviour, also observed in the solar wind at lower frequencies, is due to the Doppler effect. It can be modelled if we assume that, for the scales ranging from $k c / \omega_{p e} \simeq 0.3$ to $30\left(c / \omega_{p e}\right.$ is the electron inertial length), the intensity of the e.m. fluctuations for a wave number $k$ (i) varies like $k^{-v}$ with $v \simeq 3$, (ii) peaks for wave vectors $\boldsymbol{k}$ perpendicular to $\boldsymbol{B}$ like $\left|\sin \theta_{k B}\right|^{\mu}$ with $\mu \simeq 100$. The shape of the observed variations of $\delta B^{2}$ with $f$ and with $\Theta_{B V}$ implies that the permanent fluctuations, at these scales, statistically do not obey the dispersion relation for fast/whistler waves or for kinetic Alfvén waves: the fluctuations have a vanishing frequency in the plasma frame, i.e. their phase velocity is negligible with respect to $V$ (Taylor hypothesis). The electrostatic waves around $1 \mathrm{kHz}$ behave differently: $\delta E^{2}$ is minimum for $\Theta_{B V} \simeq 90^{\circ}$. This can be modelled, still with the Doppler effect, if we assume that, for the scales ranging from $k \lambda_{D e} \simeq 0.1$ to $1\left(\lambda_{D e}\right.$ is the Debye length), the intensity of the e.s. fluctuations (i) varies like $k^{-v}$ with $v \simeq 4$, (ii) peaks for $\boldsymbol{k}$ parallel to $\boldsymbol{B}$ like $\left|\cos \theta_{k B}\right|^{\mu}$ with $\mu \simeq 100$. These e.s. fluctuations may have a vanishing frequency in the plasma frame, or may be ion acoustic waves. Our observations imply that the e.m. frequencies observed

Correspondence to: C. Lacombe

(Catherine.Lacombe@obspm.fr)
Keywords. Magnetospheric physics (Magnetosheath; Plasma waves and instabilities) - Space plasma physics (Turbulence)

\section{Introduction}

The magnetic and electric fluctuations in the Earth's magnetosheath have been mainly studied either in the Ultra Low Frequency range (ULF, $f<10 \mathrm{~Hz}$ ) or at much higher frequencies $f \geq 1 \mathrm{kHz}$ (see the review by Lucek et al., 2005).

The intermediate range $(10 \mathrm{~Hz} \leq f \leq 1 \mathrm{kHz})$ has been given less attention, although results have been obtained thanks to spacecraft which observed electromagnetic (e.m.) and/or electrostatic (e.s.) waves in the magnetosheath. According to these results, the wave intensity in the e.m. range is mainly controlled by the position in the magnetosheath, in particular the distance of the magnetopause (Rodriguez, 1985). In the e.s. range, the wave intensity depends on the distance of the bow shock (Rodriguez, 1979); and it depends strongly on the local angle between the magnetic field $\boldsymbol{B}$ and the flow velocity $\boldsymbol{V}$ (Coroniti et al., 1994). The STAFF Spectral Analyser (STAFF-SA) on board Cluster allows one to analyse this intermediate range between $8 \mathrm{~Hz}$ and $4 \mathrm{kHz}$, i.e. between about $f_{l h}$ and $10 f_{c e}$ or $10 f_{p i}\left(f_{l h}, f_{c e}\right.$ and $f_{p i}$ are the nominal lower hybrid frequency, electron gyrofrequency and proton plasma frequency in the magnetosheath plasma rest frame).

Published by Copernicus GmbH on behalf of the European Geosciences Union. 
In the present paper (Paper 1, and in a companion Paper 2 by Lacombe et al., 2006) we show results obtained from the STAFF-SA data, the most striking of which, discussed in Paper 1, being the strong dependence of the intensity of the fluctuations, both electromagnetic and electrostatic, on the angle $\Theta_{B V}$ between the magnetic field $\boldsymbol{B}$ and the flow velocity $\boldsymbol{V}$. In Paper 1, we show that a simple interpretation of this observation is that the fluctuations have a highly anisotropic distribution of wave vectors, while their observed frequencies are mainly due to a Doppler shift. In Paper 2, we will show that no parameter other than $\Theta_{B V}$ appears to play a significant role in the turbulence intensity in the magnetosheath. Actually, the fluctuations in the STAFF-SA frequency range are made of two components, one component permanently observed, the "permanent" component, over which are superposed intermittent, short duration bursts of whistler or electrostatic waves. In both papers, we neglect the whistler waves or the electrostatic pulses, which are not always present, and we only consider the underlying permanent fluctuations.

To interpret spectral observations, the frequencies measured on board a spacecraft must be transformed to frequencies in the plasma rest frame and, if possible, to wave numbers. Any observed frequency $\omega$ can be considered as the sum of the frequency $\omega_{0}$ of a wave in the plasma rest frame plus the Doppler shift, $\omega=\omega_{0}+\boldsymbol{k} . \boldsymbol{V}$, where $\boldsymbol{k}$ is the wave vector . The Taylor hypothesis, usually made in the solar wind, implies that $\omega_{0}$ is vanishing i.e. that the phase speed of the wave $\omega_{0} / k$ is much smaller than $V$ in a large range of frequencies. If we make the Taylor hypothesis in the magnetosheath, the STAFF-SA frequencies $8 \mathrm{~Hz}$ to $4 \mathrm{kHz}$ correspond to the electron scales $\sim 10 c / \omega_{p e}$ to $\sim 3 \lambda_{D e}\left(c / \omega_{p e}\right.$ is the electron inertial length, $\lambda_{D e}$ the electron Debye length). In the present paper, we thus study the anisotropies of the wave vector distributions at electron scales (about $50 \mathrm{~km}$ to $30 \mathrm{~m}$ ) which are smaller than the Cluster separation.

In the ULF range $(f<10 \mathrm{~Hz})$ what are the directions of the wave vectors found for case studies in the magnetosheath? For the Alfvén ion cyclotron waves, the wave vectors are generally parallel to the magnetic field $\boldsymbol{B}$ (e.g. Lacombe et al., 1995; Alexandrova et al., 2004). Some observations of mirror modes show that they are 3-D structures, with the minor axis nearly along the magnetopause normal (Hubert et al., 1998) and perpendicular to both $\boldsymbol{B}$ and $\boldsymbol{V}$ (Lucek et al., 2001). The normals of the mirror structures observed by Horbury et al. (2004) suggest that they are cylinders rather than sheets. A case study with the k-filtering method, in mirrorlike fluctuations near the magnetopause, displays wave vectors mainly perpendicular to $\boldsymbol{B}$ (Sahraoui et al., 2004) and also perpendicular to the magnetopause normal (Sahraoui et al., 2006). With the same method, during 37 intervals over 5 months, Schäfer et al. (2005) find standing mirror modes with wave vectors mainly perpendicular to $\boldsymbol{B}$, and Alfvénic fluctuations at every angle with respect to $\boldsymbol{B}$; but quasi-perpendicular, mirror-like waves are also found, with phase speeds up to the local Alfvén velocity.
Above $1 \mathrm{kHz}$, the fluctuations are electrostatic: the electric field and the wave vectors are mainly parallel to $\boldsymbol{B}$. This broadband electrostatic noise is made of bipolar and tripolar pulses observed up to $f_{p e}$ with a typical duration of $0.1 \mathrm{~ms}$ (Pickett et al., 2005), superimposed on a background of waves, up to a few $\mathrm{kHz}$ (Pickett et al., 2003).

In the intermediate frequency range of STAFF-SA $(8 \mathrm{~Hz}-$ $4 \mathrm{kHz}$ ), the magnetic and the electric fluctuations at a given frequency are nearly isotropic; but we find that their total intensities $\delta B^{2}$ (in the three directions) and $\delta E^{2}$ depend strongly on the angle $\Theta_{B V}$ between $\boldsymbol{B}$ and $\boldsymbol{V}$ (Sect. 3). These strong dependences can be modelled if the $\boldsymbol{k}$ distributions $I(\boldsymbol{k})$ have a power law dependence $k^{-v}$ and if the angular distribution of $I(\boldsymbol{k})$, assumed to be axisymmetric around $B$, is highly anisotropic (Sect. 4). In the electromagnetic or "whistler" range ( $k c / \omega_{p e} \sim 0.3$ to 30 ), we find that $I(\boldsymbol{k})$ has to peak for $\boldsymbol{k}$ mostly perpendicular to $\boldsymbol{B}$, and that the permanent fluctuations statistically have a vanishing frequency in the plasma rest frame (Sect. 5). In the electrostatic or "ion acoustic" range ( $k c / \omega_{p e} \sim 15$ to 150 , or $k \lambda_{D e} \sim 0.1$ to 1$)$, the distribution $I(\boldsymbol{k})$ has to peak for $\boldsymbol{k}$ mostly parallel to $\boldsymbol{B}$; the fluctuations may have a vanishing frequency in the plasma frame, but the dispersion relation of ion acoustic waves is also statistically consistent with the observations (Sect. 6). In Sect. 7, we discuss a possible wave mode identification based on the ratio $\delta E^{2} / \delta B^{2}$ observed in the electromagnetic range. We compare our results about the anisotropies of the wave vector distributions at electron scales, in the magnetosheath, to the anisotropies observed in the solar wind. Finally, we show that the strong dependence of the intensity of the permanent e.m. and e.s. fluctuations on the angle $\Theta_{B V}$ is probably not due to the dissipation of an energy input in the magnetosphere frame.

\section{Data}

The STAFF Spectral Analyser (Cornilleau-Wehrlin et al., 1997) measures every second the diagonal terms of the $5 \times 5$ complex spectral matrix computed with the three $\delta \boldsymbol{B}$ components of the magnetic field fluctuations and two $\boldsymbol{\delta} \boldsymbol{E}$ components of the electric field fluctuations. STAFF-SA operates at 27 logarithmically spaced frequencies, between $8 \mathrm{~Hz}$ and $4 \mathrm{kHz}$. We shall use here 4-s averages of the magnetic Power Spectral Density (PSD) which is the trace of the magnetic field spectral matrix $\delta B^{2}=\delta B_{x x}^{2}+\delta B_{y y}^{2}+\delta B_{z z}^{2}$ in $\mathrm{nT}^{2} / \mathrm{Hz}$, and 4-s averages of the electric PSD $\delta E^{2}=\delta E_{x x}^{2}+\delta E_{y y}^{2}$ in $(\mathrm{mV} / \mathrm{m})^{2} / \mathrm{Hz}$. We shall also consider the phase differences between the three components of $\delta \boldsymbol{B}$, given by the nondiagonal terms of the complex spectral matrix measured every $4 \mathrm{~s}$. This 4 -s complex spectral matrix is projected in a magnetic-field aligned frame, so that $\delta B_{1}$ and $\delta B_{2}$ are the two components of $\boldsymbol{\delta} \boldsymbol{B}$ perpendicular to the $\boldsymbol{B}$ field averaged over $4 \mathrm{~s}$ : $\delta B_{1}$ is in the plane $\left(\boldsymbol{B}, \boldsymbol{X}_{\mathrm{GSE}}\right)$, with a positive component along $\boldsymbol{X}_{\mathrm{GSE}}$. A phase difference of $90^{\circ}$ between $\delta B_{1}$ 
Table 1. Coordinates of the four time intervals on Cluster-1.

\begin{tabular}{ccccccccc}
\hline date & \multicolumn{2}{c}{12.02 .2001} & \multicolumn{2}{c}{16.12 .2001} & \multicolumn{2}{c}{19.12 .2001} & \multicolumn{2}{c}{17.05 .2002} \\
\hline time (UT) & $00: 15$ & $07: 30$ & $03: 15$ & $09: 00$ & $00: 00$ & $06: 00$ & $08: 00$ & $12: 15$ \\
\hline$X R_{E}$ & 4.20 & 11.81 & -1.10 & 0.79 & 3.40 & 5.06 & 5.53 & 6.10 \\
$Y R_{E}$ & 5.04 & 7.07 & 9.97 & 15.01 & 17.74 & 18.53 & -8.69 & -12.84 \\
$Z R_{E}$ & 8.91 & 8.52 & 8.62 & 6.90 & 4.03 & 0.64 & 8.00 & 6.66 \\
LT (hour) & $14: 39$ & $14: 04$ & $18: 25$ & $17: 48$ & $17: 17$ & $16: 59$ & $08: 10$ & $07: 42$ \\
lat (deg.) & 53.6 & 31.8 & 40.7 & 24.7 & 12.6 & 1.9 & 37.8 & 25.1 \\
\hline
\end{tabular}

and $\delta B_{2}$ implies a circular right-handed polarisation, while a phase difference of $0^{\circ}$ or $180^{\circ}$ implies a linear polarisation.

We analyse four intervals of Cluster data (spacecraft 1 ), lasting from $4 \mathrm{~h}$ to $7 \mathrm{~h}$. Table 1 gives the dates, the GSE coordinates, the local time and the latitude of Cluster 1 at the beginning and the end of each interval. The considered intervals are generally far from the bow shock, except the longest interval, on 12 February 2001, which corresponds to a complete crossing of the magnetosheath. We shall mainly analyse 19 December 2001, when the Cluster spacecraft were in the dusk side.

The plasma properties, proton density, temperature and velocity (Rème et al., 1997) and the electron temperature (Johnstone et al., 1997) are sampled with a time resolution of $4 \mathrm{~s}$. We use 4-s averages of the magnetic field (Balogh et al., 1997).

\section{Observations}

The point we want to stress in this paper is the remarkable and strong dependence of $\delta B^{2}$ and $\delta E^{2}$ on the angle $\Theta_{B V}$ between the magnetic field $\boldsymbol{B}$ and the flow velocity $\boldsymbol{V}$, when observed at a given frequency in the STAFF-SA range. This strong dependence is observed while the variance of the fluctuations is nearly isotropic, so that $\delta B_{x x}^{2} \simeq \delta B_{y y}^{2} \simeq \delta B_{z z}^{2}$ and $\delta E_{x x}^{2} \simeq \delta E_{y y}^{2}$.

Let us first consider the magnetic fluctuations. Figure 1 displays scatter plots of $\delta B^{2}$ as a function of $\Theta_{B V}$ on different days and at different frequencies, the broken line being the median value for bins $5^{\circ}$ wide. Despite the scatter of the data, Fig. 1a, at $8.8 \mathrm{~Hz}$, shows that the median line displays a broad peak for $\Theta_{B V} \simeq 90^{\circ}$. On the same day, 19 December 2001, Fig. 1b displays a weaker curvature at a higher frequency, but this is due to the background noise: the background level is about $5 \times 10^{-9} \mathrm{nT}^{2} / \mathrm{Hz}$ at $56 \mathrm{~Hz}$ (CornilleauWehrlin et al., 2003), and it prevents the measurements of weaker signals for $\Theta_{B V}<50^{\circ}$. Figure 1c shows that $\Theta_{B V}$ varies over about $180^{\circ}$ in $4 \mathrm{~h}$ on 17 May 2002: it helps to see that there is probably a symmetry between $\Theta_{B V}<90^{\circ}$ and $\Theta_{B V}>90^{\circ}$. On 16 December $2001, \delta B^{2}$ is very intense, well above the background noise: there is a broad peak of
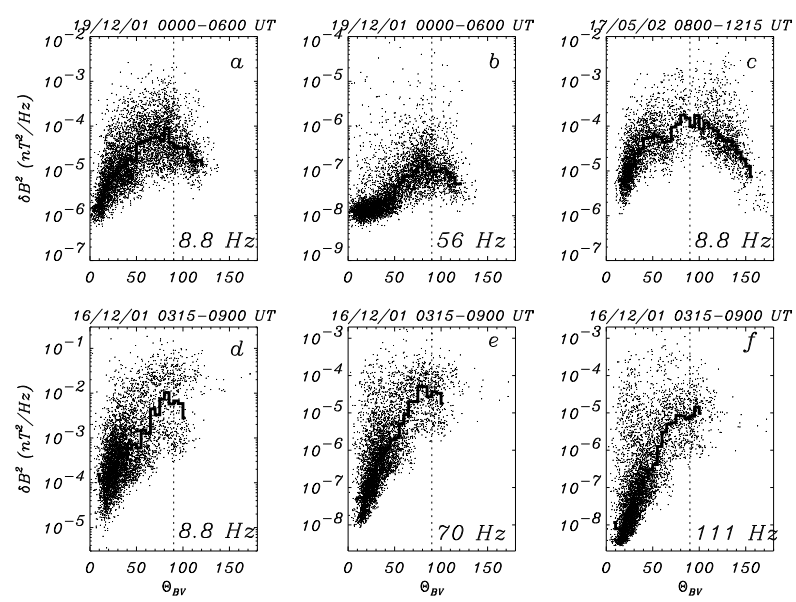

Fig. 1. Scatter plots of the trace $\delta B^{2}$ of the spectral matrix of the magnetic fluctuations every $4 \mathrm{~s}$, as a function of the angle $\Theta_{B V}$ between the $\boldsymbol{B}$ field and the flow velocity $\boldsymbol{V}$ in the magnetosheath: (a)19 December 2001, for $f=8.8 \mathrm{~Hz}$; (b) 19 December 2001, $f=56 \mathrm{~Hz}$; (c) 17 May 2002, $8.8 \mathrm{~Hz}$; (d) 16 December 2001, $8.8 \mathrm{~Hz}$; (e) 16 December 2001, $70 \mathrm{~Hz}$; (f) 16 December 2001, $111 \mathrm{~Hz}$. The thick line gives the median value for bins $5^{\circ}$ wide.

the median line for $\Theta_{B V} \simeq 90^{\circ}$ and a strong curvature of the scatter plot at $8.8 \mathrm{~Hz}$ (Fig. 1d), as well as at $70 \mathrm{~Hz}$ and $111 \mathrm{~Hz}$ (Figs. 1e and 1f).

As mentionned in the Introduction, the fluctuating fields are made of several components. This is clearly shown by histograms of the PSD $\delta B^{2}$ at a given frequency, and histograms of the phase difference $\phi_{B 1 B 2}$ between the two components $\delta B_{1}$ and $\delta B_{2}$ perpendicular to the local $\boldsymbol{B}$ field (Fig. 2). In the histogram of Fig. 2a, the solid line corresponds to the data of the scatter plot of Fig. 1a: this is a nearly Gaussian distribution, at $8.8 \mathrm{~Hz}$. The corresponding phases $\phi_{B 1 B 2}$ (Fig. 2b) are around $0^{\circ}$ and $180^{\circ}$ and imply a linear polarisation. Whistler waves, which are right-handed, should appear as a peak at $\phi_{B 1 B 2} \simeq 90^{\circ}$, if they were intense enough. The dotted line in Fig. 2a gives the histogram of the PSD of the fluctuations with $\phi_{B 1 B 2}=90^{\circ} \pm 10^{\circ}$ : these whistler waves have a negligible power at $8.8 \mathrm{~Hz}$. The histogram (solid line) of Fig. 2c corresponds to the data of Fig. 1e at a higher 

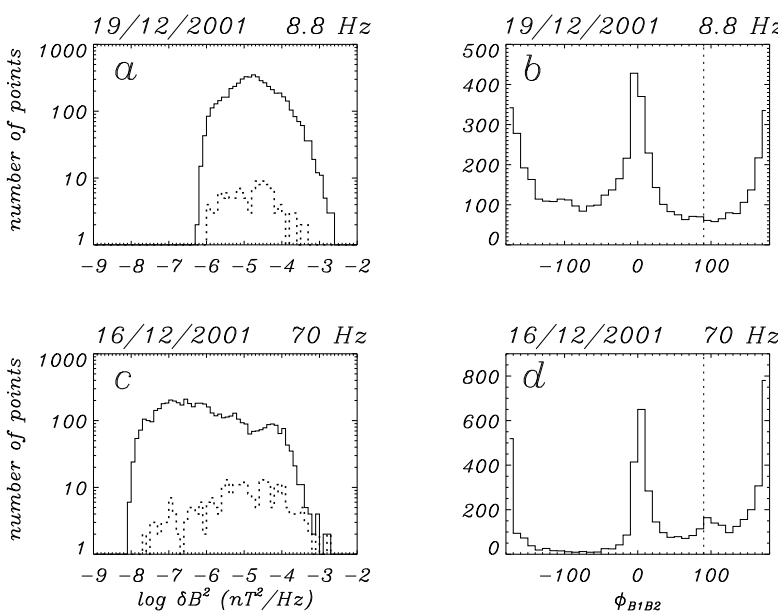

Fig. 2. 19 December 2001 at $8.8 \mathrm{~Hz}$ : (a) histograms of $\log \delta B^{2}$ $\left(\mathrm{nT}^{2} / \mathrm{Hz}\right)$ for the whole data set (solid line), and for the right-handed fluctuations (dotted line); (b) histogram of the phase difference $\phi_{B 1 B 2}$ between the two components $\delta B_{1}$ and $\delta B_{2}$ of the fluctuations perpendicular to the local $\boldsymbol{B}$ field (4 s average). 16 December 2001 at $70 \mathrm{~Hz}$ : (c) histogram of $\log \delta B^{2}$ for the whole data set (solid line), and for the right-handed fluctuations (dotted line); (d) histogram of the phase difference $\phi_{B 1 B 2}$.

frequency $(70 \mathrm{~Hz})$ : on the Gaussian distribution is superposed a shoulder of less frequent and more intense fluctuations. The corresponding phases (Fig. 2d) are still mainly linear $\left(\phi_{B 1 B 2}=0^{\circ}\right.$ or $\left.180^{\circ}\right)$ but a few whistler waves are present with a right-handed polarisation $\phi_{B 1 B 2} \simeq 90^{\circ}$. In Fig. 2c, the dotted line gives the histogram of the PSD of these whistlers: at $70 \mathrm{~Hz}$, the whistlers are relatively more important above $10^{-5} \mathrm{nT}^{2} / \mathrm{Hz}$ than below. They are not dominant but they contribute to the dispersion of the scatter plots of Figs. 1e and $1 \mathrm{f}$. The low intensity boundary of each scatter plot has a maximum for $\Theta_{B V} \simeq 90^{\circ}$ : this is typical of the permanent e.m. turbulence. The high intensity boundary of each scatter plot has no clear maximum: this is due to whistler waves which will be analysed in a future work. The time intervals with whistler waves at different frequencies have not been withdrawn from the data because the whistlers are relatively rare and because their occurrence and their intensity do not depend on $\Theta_{B V}$. The scatter plots and the medians of Fig. 1 thus mainly correspond to permanent fluctuations with a Gaussian histogram, which are the subject of our study.

In Fig. 3, we show the spectra averaged over several hours on the four considered days, for large angles $\left(65^{\circ}<\Theta_{B V}<115^{\circ}\right.$, solid lines) and for small angles $\left(\Theta_{B V}<25^{\circ}\right.$ and $\Theta_{B V}>155^{\circ}$, dashed lines). From $f_{l h}$ to $f_{c e}, \delta B^{2}$ is always more intense for large $\Theta_{B V}$. The dotted line gives the observed minimum PSD over each interval, which is near the sensitivity of STAFF-SA given by Fig. 2 of Cornilleau-Wehrlin et al. (2003). The spectral bumps around $70 \mathrm{~Hz}$ on Figs. $3 \mathrm{a}$ and $3 \mathrm{~d}$ are observed for large and small
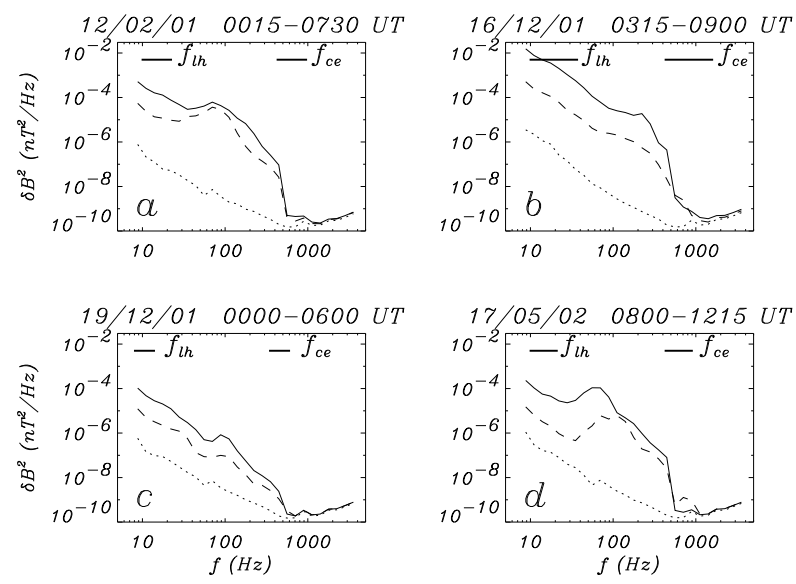

Fig. 3. Average power spectral density $\delta B^{2}(f)$ for large angles $\left(65^{\circ}<\Theta_{B V}<115^{\circ}\right.$, solid line $)$ and for small angles $\left(\Theta_{B V}<25^{\circ}\right.$, $\Theta_{B V}>155^{\circ}$, dashed line), for the four considered intervals. The dotted line gives the observed minimum PSD over each interval. The horizontal bars at the top of each figure give the range of lower hybrid frequencies $f_{l h}$ and of electron cyclotron frequencies $f_{c e}$ found during the interval.

$\Theta_{B V}$; they are due to whistlers which are relatively more frequent or more intense on 12 February 2001 and on 17 May 2002 than on the two other days. The scales $k^{-1}=V / 2 \pi f$ corresponding to $8 \mathrm{~Hz}-800 \mathrm{~Hz}$ give $k c / \omega_{p e} \simeq 0.3$ to 30 , so that the wavelengths are $\simeq 40 \mathrm{~km}$ to $400 \mathrm{~m}$, smaller than the separation between the Cluster spacecraft. The spectral slope is $v \simeq 3$ around 10 to $30 \mathrm{~Hz}$, and $v \simeq 4$ above $100 \mathrm{~Hz}$ (Fig. 3).

Let us now consider the electric fluctuations. As we did not withdraw the whistlers from the magnetic fluctuations, we do not withdraw them from the electric fluctuations in the electromagnetic range (below $\simeq 300 \mathrm{~Hz}$ ) because they are not dominant. In the electrostatic range (above $\simeq 300 \mathrm{~Hz}$ ) the short duration pulses observed in the time domain by Pickett et al. (2005) probably do not play a large part in our data which are 4-s averages of the PSD. We have not tried to withdraw them from our data: indeed, Pickett et al. (2005) note that neither the time duration nor the amplitude of the pulses depend on $\Theta_{B V}$. Conversely, Coroniti et al. (1994) noted that, around $1 \mathrm{kHz}$ in the magnetosheath, $\delta E^{2}$ is large for small $\Theta_{B V}$ and vanishes for $\Theta_{B V} \simeq 90^{\circ}$. We also observe that the electric PSD at a given frequency depends on the angle $\Theta_{B V}$, but with a change in regime between low and high frequencies. Indeed, the spectra of Fig. 4 show that $\delta E^{2}$ is more intense for large $\Theta_{B V}$ (solid lines) below a frequency $f_{r} \simeq 200$ to $1000 \mathrm{~Hz}$, while $\delta E^{2}$ is more intense for small $\Theta_{B V}$ (dashed lines) above $f_{r} ; f_{r}$ is slightly below $f_{p i}$, and is below or around $f_{c e}$. Figures $5 \mathrm{a}$ to 5e display $\delta E^{2}$ as a function of $\Theta_{B V}$ on day 19 December 2001, at different frequencies. At $8.8 \mathrm{~Hz}$ (Fig. 5a) there is no maximum of $\delta E^{2}$ for $\Theta_{B V} \simeq 90^{\circ}$, while the maximum of 

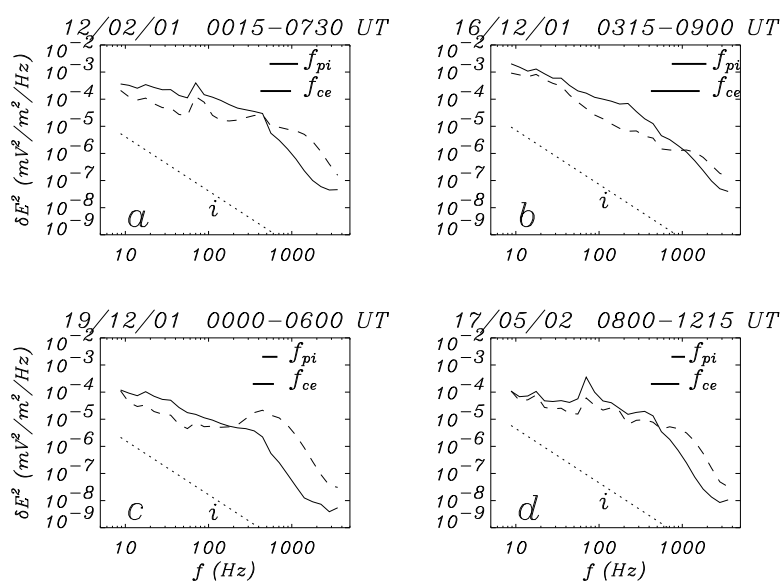

Fig. 4. Average power spectral density $\delta E^{2}(f)$ for large angles $\left(65^{\circ}<\Theta_{B V}<115^{\circ}\right.$, solid line $)$ and for small angles $\left(\Theta_{B V}<25^{\circ}\right.$, $\Theta_{B V}>155^{\circ}$, dashed lines), for the four considered intervals. The dotted line $i$ gives an estimation of the background impact noise on the antenna, which varies like $f^{-2}$. The horizontal bars at the top of each figure give the range of proton plasma frequencies $f_{p i}$ and of electron cyclotron frequencies $f_{c e}$ found during the interval.

$\delta B^{2}$ at the same frequency was clear (Fig. 1a). But at $18 \mathrm{~Hz}$ (Fig. 5b) and at $88 \mathrm{~Hz}$ (Fig. 5c), $\delta E^{2}$ has a broad maximum for $\Theta_{B V} \simeq 90^{\circ}$. At higher frequencies, there is a relative minimum for $\Theta_{B V} \simeq 90^{\circ}$ at $445 \mathrm{~Hz}$ (Fig. 5d), and a deeper minimum at $891 \mathrm{~Hz}$ (Fig. 5e). In Fig. 5e, the dispersion of the data $\delta E^{2}$ at $\Theta_{B V} \simeq 20^{\circ}$ is larger than at $\Theta_{B V} \simeq 90^{\circ}$. Note that Fig. 5f (day 17 May 2002) displays a scatter plot which is not symmetrical with respect to $\Theta_{B V}=90^{\circ}$. This point will be addressed in Sect. 7.2. The spectral slope of $\delta E^{2}$ varies from $v \simeq 1$ to 2 below $f_{p i}$, and is about 4 above $f_{p i}$ (Fig. 4 ). In the e.m. range, below $f_{p i}$, the spectral slope of $\delta E^{2}$ is thus weaker than the spectral slope of $\delta B^{2}$. We shall see in Sect. 6 how these different behaviours for $\delta E^{2}\left(f, \Theta_{B V}\right)$ can be modelled.

\section{Models of the anisotropic distribution of wave vectors of the turbulence}

A possible explanation for the dependence on $\Theta_{B V}$ of the levels $\delta B^{2}$ and $\delta E^{2}$ of the permanent fluctuations, at a given observing frequency, is that the observations are affected by a significant Doppler effect. Indeed, a natural assumption is that the intensity of the permanent turbulence increases with a decreasing wave number $k$. A given Doppler shift $2 \pi f=k V \cos \theta_{k V}$ will be reached by a small $k$ (which has a large intensity), if $\cos \theta_{k V}$ is large $\left(\theta_{k V} \simeq 0^{\circ}\right)$ : this happens for $\Theta_{B V} \simeq 0^{\circ}$, if $\boldsymbol{k}$ is mostly parallel to $\boldsymbol{B}$, and for $\Theta_{B V} \simeq 90^{\circ}$, if $\boldsymbol{k}$ is mostly perpendicular to $\boldsymbol{B}$. $\theta_{k V}$ is the angle between $\boldsymbol{k}$ and $\boldsymbol{V}$.
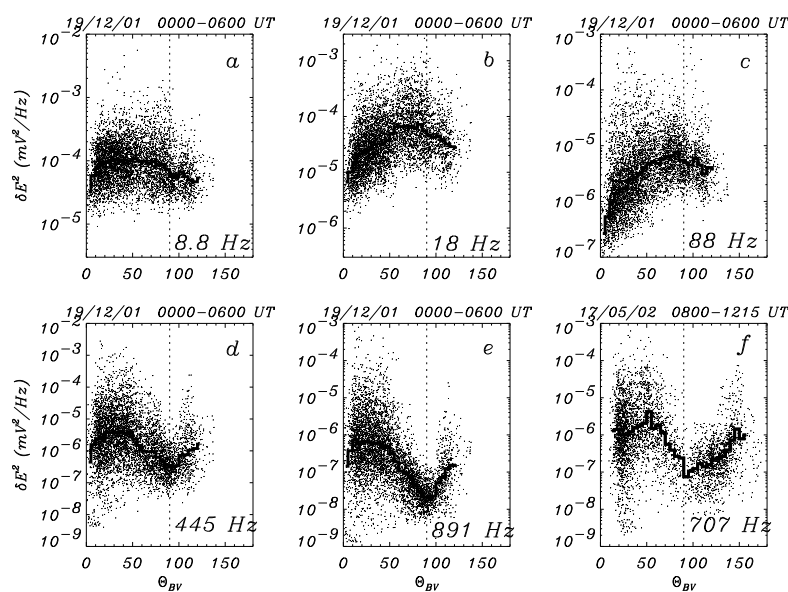

Fig. 5. Scatter plots of $\delta E^{2}$ as a function of the angle $\Theta_{B V}$. 19 December 2001: (a) at $f=8.8 \mathrm{~Hz}$; (b) $18 \mathrm{~Hz}$; (c) $88 \mathrm{~Hz}$; (d) $445 \mathrm{~Hz}$; (e) $891 \mathrm{~Hz} .17$ May 2002: (f) $707 \mathrm{~Hz}$. The thick line gives the median value for bins $5^{\circ}$ wide.

We shall explore the possibility that the Doppler effect, combined with an anisotropic distribution of wave vectors, explains all or a major part of the observed PSD variations: simple models with reasonable properties naturally account for the observations.

We shall see in Sect. 7.4 that the dependence of $\delta B^{2}$ and $\delta E^{2}$ on $\Theta_{B V}$ in the magnetosheath is probably not due to the dissipation of an energy input like the solar wind Poynting vector energy flux $\boldsymbol{E}_{S W} \times \boldsymbol{B}_{S W}$ in the magnetosheath.

\subsection{General method}

A 3-D wave vector spectrum $I_{3 D}(\boldsymbol{k})$ of the magnetosheath fluctuations cannot be directly measured with the STAFF Spectral Analysers, as the information on phase delays between the four probes is lost for wavelengths smaller than the separation. However, as usual in space physics, the motion of the plasma with respect to one probe allows a 1-D analysis of the wave vector spectrum, along the direction $\boldsymbol{V}$ of the flow velocity.

Let us assume that $I_{3 D}(\boldsymbol{k})$ is axisymmetric with respect to the direction of the mean $\boldsymbol{B}$ field, so that it only depends on two parameters, $k$ and the angle $\theta_{k B}$ between $\boldsymbol{k}$ and $\boldsymbol{B}$. Then, if the angle $\Theta_{B V}$ between $\boldsymbol{B}$ and $\boldsymbol{V}$ changes, the other plasma parameters remaining roughly constant, $I_{3 D}\left(k, \theta_{k B}\right)$ is sampled in different directions $\theta_{k B}$.

We use a coordinate system with the $x$ axis aligned with $\boldsymbol{V}$, and the $\boldsymbol{B}$ field in the $x, y$ plane. If $\omega_{0}$ is the frequency of a wave in the plasma frame ( $\omega_{0}$ is assumed to be positive), the observed frequency is

$\omega=\left|k_{x} V+\omega_{0}\left(k, \theta_{k B}\right)\right|$, 
where $k_{x}$ can be $>0$ or $<0$. The angle $\theta_{k B}$ can be written as a function of $\Theta_{B V}$,

$$
\sin \theta_{k B}=\left[k_{z}^{2}+\left(k_{x} \sin \Theta_{B V}-k_{y} \cos \Theta_{B V}\right)^{2}\right]^{1 / 2} / k,
$$

where $\theta_{k B}$ and $\Theta_{B V}$ vary between 0 and $\pi$. We introduce the function

$h\left(k_{x}\right) \equiv \omega-\left|k_{x} V+\omega_{0}\left(k, \theta_{k B}\right)\right|$,

so that Eq. (1) is equivalent to $h\left(k_{x}\right)=0$. The power $\delta B^{2}\left(\omega, \Theta_{B V}\right)$ (or $\delta E^{2}\left(\omega, \Theta_{B V}\right)$ ) observed at a given frequency for a given angle can be written as the sum of contributions at different scales $k^{-1}$

$\delta B^{2}\left(\omega, \Theta_{B V}\right)=A \int_{-\infty}^{\infty} d k_{y} \int_{-\infty}^{\infty} d k_{z} \int_{-\infty}^{\infty} d k_{x} \delta\left[h\left(k_{x}\right)\right] I_{3 D}(\boldsymbol{k})$

where $A$ is a normalisation factor and $\delta(x)$ the usual Dirac function. There are generally several solutions $k_{x s}$ (positive or negative) to the equation $h\left(k_{x}\right)=0$ (argument of the Dirac function). $\theta_{k B}$ is a function of $k_{x s}, k_{y}, k_{z}$ and $\Theta_{B V}$ (Eq. 2). After the integration over $k_{x}$, we obtain

$$
\begin{aligned}
& \delta B^{2}\left(\omega, \Theta_{B V}\right)= \\
& \quad A \Sigma_{\mathbf{s}} \int_{-\infty}^{\infty} d k_{y} \int_{-\infty}^{\infty} d k_{z} I_{3 D}\left(k_{x s}, k_{y}, k_{z}\right) /\left|\frac{d h}{d k_{x}}\left(k_{x s}\right)\right|
\end{aligned}
$$

where $\Sigma_{s}$ is a sum over the $k_{x s}$ solutions. In what follows, we shall make simple assumptions about the dependence of $I_{3 D}\left(k, \theta_{k B}\right)$ on $k$ and $\theta_{k B}$ :

- $I_{3 D}$ has a power law dependence on the wave number

$$
I_{3 D} \propto k^{-v-2}
$$

in a range $k_{\min }<k<k_{\max }$, with a spectral index $v$ independent on $\theta_{k B}$,

- for a given $k, I_{3 D}$ has one of the two typical angular distributions,

$$
I_{3 D} \propto\left|\cos \theta_{k B}\right|^{\mu}
$$

illustrating situations when $\boldsymbol{k}$ lies mostly parallel to $B$, and

$$
I_{3 D} \propto\left|\sin \theta_{k B}\right|^{\mu}
$$

for $\boldsymbol{k}$ mostly perpendicular to $B$.

Note that the exponent $v$ refers here to the power law index of the 1-D spectrum $I_{1 D}(k)$ defined by

$\delta B^{2}=\int d k I_{1 D}(k)$

with

$I_{1 D}(k)=2 \pi A k^{2} \int_{0}^{\pi} \sin \theta_{k B} d \theta_{k B} I_{3 D}\left(k, \theta_{k B}\right)$.

For an isotropic Kolmogorov spectrum $I_{1 D} \propto k^{-v}$ with $\nu=5 / 3$, the 3 -D spectrum is $I_{3 D} \propto k^{-\nu-2}$.

\subsection{Parameters of the models}

Our aim is to study the respective influence on $\delta B^{2}\left(\omega, \Theta_{B V}\right)$ and on $\delta E^{2}\left(\omega, \Theta_{B V}\right)$ of the anisotropy of the $k$ distribution, of the Doppler shift and of possible dispersion effects. We shall first test whether simple anisotropic models for $I_{3 D}\left(k, \theta_{k B}\right)$ (Eq. 5, with Eq. 6 or Eq. 7) can explain the observed behaviour of $\delta B^{2}\left(f, \Theta_{B V}\right)$ and $\delta E^{2}\left(f, \Theta_{B V}\right)$ described in Sect. 3.

To compare models and observations, we shall mainly consider 19 December 2001 because the variations of the plasma parameters, velocity, density, temperatures and magnetic field are only $20 \%$ to $35 \%$ over $6 \mathrm{~h}$. (Conversely, on 16 December 2001 the proton density, for instance, varies from 10 to $70 \mathrm{~cm}^{-3}$ over $6 \mathrm{~h}$; see Paper 2). On 19 December 2001, the average parameters are $V=260 \mathrm{~km} / \mathrm{s}$ for the flow speed, $148.4 \mathrm{~km} / \mathrm{s}$ for the Alfvén speed $v_{A}, 152.7 \mathrm{~km} / \mathrm{s}$ for the sound speed $c_{s}=\left(\gamma k_{B}\left(T_{e}+T_{p}\right) / m_{p}\right)^{1 / 2}(\gamma=5 / 3$ is the ratio of specific heats), so that $c_{s}^{2} / v_{A}^{2}=1.06 ; \beta_{p}=1.07$, $\beta_{e}=0.2, c / \omega_{p e} \simeq 2 \mathrm{~km}, c / \omega_{p i} \simeq r_{g i} \simeq 90 \mathrm{~km}$, the Debye length $\lambda_{D e} \simeq 15 \mathrm{~m}, f_{p i} \simeq 530 \mathrm{~Hz}, f_{c e} \simeq 484 \mathrm{~Hz}$ and $f_{l h} \simeq 11.3 \mathrm{~Hz}$. The temperature anisotropies are $T_{p \perp} / T_{p \|}=1.65$ for the protons, and $T_{e \perp} / T_{e \|} \simeq 1$ for the electrons.

The models $\delta B^{2}\left(f, \Theta_{B V}\right)$ or $\delta E^{2}\left(f, \Theta_{B V}\right)$ (Eq. 4) will be calculated for the 27 frequencies of STAFF-SA (from $8 \mathrm{~Hz}$ to $4 \mathrm{kHz}$ ) and for 19 values of $\Theta_{B V}$ from $0^{\circ}$ to $180^{\circ}$. For the spectral index of the magnetic fluctuations, we shall take $\nu=3$ (at low frequencies) to 4 (at high frequencies), as in Fig. 3c, and $\nu=1$ to 4 for the electric fluctuations (Fig. 4c). The cone aperture of $\theta_{k B}$, corresponding to Eqs. (6 and 7), is about $20^{\circ}$ for $\mu=10$ and $7^{\circ}$ for $\mu=100$.

\section{Magnetic fluctuations in the "whistler" range}

\subsection{Models with pure Doppler effects}

First, let us assume that the Doppler shift is much larger than the wave frequency $\left(\omega_{0} \simeq 0\right)$, so that Eq.(1) reduces to $\omega-\left|k_{x} V\right|=0$. This implies that the STAFF-SA "whistler" range $(8 \mathrm{~Hz}$ to $500 \mathrm{~Hz})$ has wave numbers comparable to the inverse of the electron inertial length, $k c / \omega_{p e} \simeq 3$. We choose here $k_{\min } c / \omega_{p e}=0.3$ and $k_{\max } c / \omega_{p e}=30$. We further checked that the results do not depend on the precise values of the bounds, as soon as the $k$ domain is extended enough, covering two decades. The spectral index was chosen to be $\nu=3$ for $0.3 \leq k c / \omega_{p e} \leq 3$ and $\nu=4$ for $3 \leq k c / \omega_{p e} \leq 30$.

We first calculate $\delta B^{2}\left(f, \Theta_{B V}\right)$ (Eq. 4) for wave vectors mainly parallel to $\boldsymbol{B}$ (Eq. 6) with $\mu=10$. Figure 6a gives $\delta B^{2}$ from $f=8.8 \mathrm{~Hz}$ (upper solid line) to $56 \mathrm{~Hz}$ (lower solid line) and to $561 \mathrm{~Hz}$ (lowest dotted line); this highest frequency $561 \mathrm{~Hz}$ correspond to the largest Doppler shift $k_{\max } V$. At all the frequencies, $\delta B^{2}\left(\Theta_{B V}\right)$ is minimum for $\Theta_{B V}=90^{\circ}$. We see in Fig. 6b that the scatter plot or the median of $\delta B^{2}$ at $8.8 \mathrm{~Hz}$ cannot be explained by a model (solid line) with $\boldsymbol{k}$ 
mostly parallel to $\boldsymbol{B}$; similarly, Fig. 6 c shows that the average spectrum for small angles $\Theta_{B V}$ (s.a., defined in Sect. 3.1, dashed line) is more intense than the spectrum for large $\Theta_{B V}$ (1.a., solid line), while the opposite is observed (Fig. 3).

As discussed at the beginning of Sect. 4, we indeed expect that the wave vectors are mainly perpendicular to $\boldsymbol{B}$ in the e.m. range: we have evaluated Eq. (4) with the angular distribution of Eq. (7). To check the influence of the angular width, we consider two cases, a wide one $\left(20^{\circ}\right)$ with $\mu=10$ and a narrow one $\left(7^{\circ}\right), \mu=100$. Figure $6 \mathrm{~d}$ gives the calculated PSD $\delta B^{2}\left(f, \Theta_{B V}\right)$ for the wide angular width, for $f$ between the lowest frequency $8.8 \mathrm{~Hz}$ (upper solid line) and $561 \mathrm{~Hz}$ (lowest dotted line). At all the frequencies, the peak of $\delta B^{2}\left(\Theta_{B V}\right)$ is for $\Theta_{B V}=90^{\circ}$. Figure 6e shows that the agreement is not very good, at $8.8 \mathrm{~Hz}$, between the scatter plot or the median of the observations (in $\mathrm{nT}^{2} / \mathrm{Hz}$ ) and the model with an arbitrary normalisation factor $A=10^{-18}$ (solid line). Figure $6 \mathrm{f}$ displays the calculated spectra for large angles $\Theta_{B V}$ (solid line) and for small angles (dashed line): at every frequency, the calculated $\delta B^{2}$ is larger for large angles, as in the observed spectra (Fig. 3).

Let us now consider the narrow angular width, $\mu=100$. Then (Fig. 6h), the agreement is better between the median of the observations and the model, with $A=510^{-18}$. Figure $6 \mathrm{~g}$ shows that the curvature of $\delta B^{2}\left(\Theta_{B V}\right)$ is larger at $56 \mathrm{~Hz}$ (lower solid line) than at $8.8 \mathrm{~Hz}$. In Fig. 6i, the ratio between the 1.a. spectrum and the s.a. spectrum is larger than 10, and this is more consistent with the observations (Fig. 3).

The shape of the spectra in Figs. 6c, 6f and 6i is $f^{-v}$ with $\nu=3$ at low frequencies and $\nu=4$ at high frequencies: in models with pure Doppler effects $(f=\boldsymbol{k} . \boldsymbol{V} / 2 \pi)$ the observed spectral index $v$ of the frequency spectrum is equal to the spectral index of the 1-D wave number spectrum, regardless of the anisotropy of the (axisymmetric) wave vector distribution.

In the "whistler" range, the shape of the Power Spectral Density $\delta B^{2}$ observed as a function of $f$ and $\Theta_{B V}$ can therefore be explained by the Doppler shift of fluctuations with a vanishing rest frame frequency. The wave vector distribution has to be strongly anisotropic, with (i) a spectral density peaking for $\boldsymbol{k}$ perpendicular to $\boldsymbol{B}$ like $\left|\sin \theta_{k B}\right|^{100}$, and (ii) a steep power law dependence in $k$, like $k^{-3}$.

\subsection{Effects of a nonvanishing rest frame frequency}

Let us now assume that the rest frame frequency $\omega_{0}(\boldsymbol{k})$ is comparable to the Doppler shift. Does the inclusion of $\omega_{0}(\boldsymbol{k}) \neq 0$ in $h\left(k_{x}\right)$ (Eq. 3) significantly modify the evaluation of the model PSD (Eq. 4)?

First, note that we have to extend somewhat the range of wave numbers towards small values to reach the same STAFF-SA frequencies: we shall now use the range $0.03 \leq k c / \omega_{p e} \leq 30$. Furthermore, to avoid cumbersome and time consuming numerical solutions of kinetic dispersion equations, we shall use analytical approximate solutions
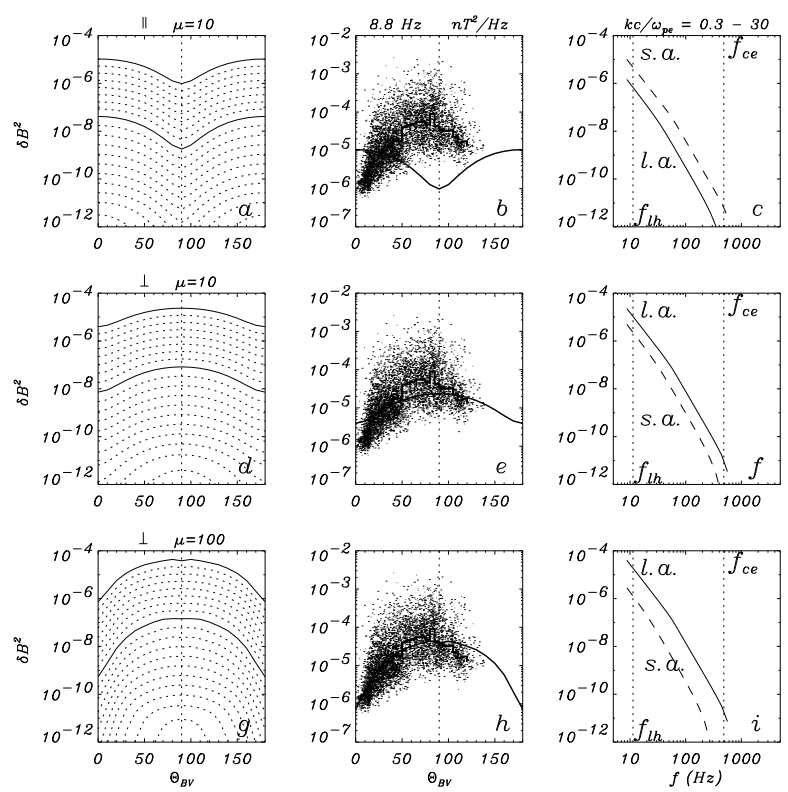

Fig. 6. The model $\delta B^{2}\left(f, \Theta_{B V}\right)$ (Eq. 4) with a pure Doppler shift and no dispersion relation in Eq. (1). The distribution $I_{3 D}(\boldsymbol{k})$ is given by Eq. (5) with $\nu=3$ to 4 , for $k c / \omega_{p e} \simeq 0.3$ to 30 , and for the average parameters of 19 December 2001. For wave vectors mostly parallel to $\boldsymbol{B}$ (Eq. 6) and for $\mu=10$ (large angular width of $\left.I_{3 D}(\boldsymbol{k})\right)$ : (a) the model $\delta B^{2}\left(\Theta_{B V}\right)$ at the STAFF-SA frequencies, from $8.8 \mathrm{~Hz}$ (upper solid line) to $561 \mathrm{~Hz}$ (lower dotted line); arbitrary ordinates. (b) the model at $8.8 \mathrm{~Hz}$ (solid line) compared to the scatter plot and the median of the observations at the same frequency, as a function of $\Theta_{B V}$; (c) the model spectra for large $\Theta_{B V}$ angles (solid line, l.a.) and for small $\Theta_{B V}$ angles (dashed line, s.a.). The vertical dotted lines give the average values of $f_{l h}$ and $f_{c e}$ on 19 December 2001. For wave vectors mostly perpendicular to $\boldsymbol{B}$ (Eq. 7) and for $\mu=10$ (large angular width of $I_{3 D}(\boldsymbol{k})$ ): (d) the model $\delta B^{2}\left(\Theta_{B V}\right)$ at different frequencies, (e) the model (solid line) and the data, still at $8.8 \mathrm{~Hz}$; (f) the model spectra. With Eq. (7) and for $\mu=100$ (stronger anisotropy of $\left.I_{3 D}(\boldsymbol{k})\right)$ : $(\mathbf{g})$ the model $\delta B^{2}\left(\Theta_{B V}\right)$ at different frequencies; (h) a good agreement between the model (solid line) and the data; (i) the model spectra.

$\omega_{0}\left(k, \theta_{k B}\right)$ of the dispersion equations. Different shapes for the dispersion relation can be found in the considered wave number range:

- modes with a phase velocity $\omega_{0} / k$ independent of $k$ and nearly independent of $\theta_{k B}$, an example being the fast MHD mode

$\omega_{0} \simeq k c_{f}$,

where $c_{f}=\left(v_{A}^{2}+c_{s}^{2}\right)^{1 / 2}$ is the fast mode velocity for perpendicular propagation; 
- modes with a phase velocity which depends mainly on $\theta_{k B}$, as in the ion acoustic mode or the slow mode

$$
\begin{aligned}
\left.\omega_{0} \simeq \sqrt{(} k_{B} T_{e} / m_{p}\right) \frac{k \cos \theta_{k B}}{\left.\sqrt{(} 1+k^{2} \lambda_{D e}^{2}\right)} \\
\\
{\left[1+3 \frac{T_{p}}{T_{e}}\left(1+k^{2} \lambda_{D e}^{2}\right)\right]^{1 / 2} \simeq k c_{s} \cos \theta_{k B} . }
\end{aligned}
$$

This dispersion relation is derived from Eqs. 10.55 and 10.113 of Baumjohann and Treumann (1996).

- modes with a phase velocity which depends on $k$ and on $\theta_{k B}$, as in the whistler mode

$$
\omega_{0} \simeq \omega_{c e} \frac{c^{2}}{\omega_{p e}^{2}} \frac{k^{2} \cos \theta_{k B}}{1+k^{2} c^{2} / \omega_{p e}^{2}},
$$

or in the Alfvén mode, taking into account kinetic effects

$\omega_{0} \simeq k v_{A} \cos \theta_{k B}\left[1+k^{2} \sin ^{2} \theta_{k B} r_{g i}^{2}\left(3 / 4+T_{e} / T_{p}\right)\right]^{1 / 2}(11)$

(see Eq. 10.179 of Baumjohann and Treumann (1996); the factor $3 / 4+T_{e} / T_{p}$ is about 1 for the average parameters of 19 December 2001).

To check the validity of these analytical dispersion relations, we calculate using the program WHAMP (Rönnmark, 1982) the fully kinetic dispersion relations for the average parameters of 19 December 2001. For a quasi-perpendicular propagation $\theta_{k B}=85^{\circ}$ and for $k c / \omega_{p e} \leq 0.3$, all the modes (mirror, Alfvén and fast) are damped; there is no whistler mode, and the fast and Alfvén mode merge with the ion Bernstein modes. For $k c / \omega_{p e} \geq 0.3$, all the modes are so strongly damped that the solutions of WHAMP are uncertain. As $T_{e} \simeq 0.2 T_{p}$ on that day, the slow mode is strongly damped for every $k$ and every $\theta_{k B}$.

We shall therefore modify our evaluation of Eq. (4), using the analytical dispersion relations described above (Eqs. 8, 9 and 11) in the calculation of $h\left(k_{x}\right)$, in spite of the fact that these modes are damped. To comply with the kinetic theory of the dispersion, only fluctuations with $k c / \omega_{p e}=0.03$ to 0.3 are assumed to be Doppler-shifted waves with a non zero rest frame frequency, while a pure Doppler shift will be considered for the range $k c / \omega_{p e}=0.3$ to 30 . (The results are not basically changed if the approximate dispersion relations are assumed to be valid in the whole range $k c / \omega_{p e}=0.03$ to 30 ). We still assume that $\nu=3$ or 4 , and $\mu=100$.

Figures $7 \mathrm{a}$ and $7 \mathrm{~b}$ display the $\operatorname{PSD} \delta B^{2}\left(f, \Theta_{B V}\right)$ obtained using the fast mode dispersion equation (Eq. 8) for a quasiperpendicular propagation, with $c_{f}=215 \mathrm{~km} / \mathrm{s}$. Figure $7 \mathrm{a}$ gives the shape of the PSD from $8.8 \mathrm{~Hz}$ (upper solid line) to $561 \mathrm{~Hz}$ (lowest dotted line). We see in Fig. 7b that if the model fits the data for $\Theta_{B V}$ larger than $30^{\circ}$, it does not fit them for $\Theta_{B V}$ smaller than $20^{\circ}$. Thus, the observed fluctuations probably do not obey the fast mode dispersion relation for a quasi-perpendicular propagation.

Figures $7 \mathrm{c}$ and $7 \mathrm{~d}$ give $\delta B^{2}\left(f, \Theta_{B V}\right)$ for the slow-ion acoustic dispersion relation (Eq. 9) for a quasi-perpendicular propagation: we take this mode into account in spite of the fact that it is strongly damped. We see in Fig. 7d that the agreement between the model and the observations at $8.8 \mathrm{~Hz}$ is as good as in Fig. 6h (pure Doppler shift), basically because the slow-ion acoustic rest frame frequency, for a quasiperpendicular propagation, remains very small compared to the Doppler shift.

Figures 7e and 7f give $\delta B^{2}\left(f, \Theta_{B V}\right)$ for the dispersion relation (Eq. 11) of quasi-perpendicular Alfvén waves, taking into account kinetic effects: there is no agreement between the model and the observations at $8.8 \mathrm{~Hz}$. Let us now assume that the wave vectors of the Alfvén waves are not quasi-perpendicular but are quasi-parallel in the range $k c / \omega_{p e}=0.03$ to 0.3 , with $I_{3 D}$ given by Eq. (6) for $\mu=100$. The intermittent presence of such waves is probable: indeed, according to WHAMP, Alfvén ion cyclotron (AIC) waves are unstable on 19 December 2001, at $k c / \omega_{p e} \simeq 0.01$, for a quasi-parallel propagation. The AIC waves are unstable, while the mirror modes are damped, because the proton temperature anisotropy is relatively large and $\beta_{p}$ relatively small on 19 December 2001 (see Lacombe and Belmont, 1995). Figures $7 \mathrm{~g}$ and $7 \mathrm{~h}$ give $\delta B^{2}\left(f, \Theta_{B V}\right)$ for quasi-parallel Doppler-shifted Alfvén waves (in the range $k c / \omega_{p e}=0.03$ to 0.3 ), and for a pure Doppler shift of quasiperpendicular fluctuations (in the range $k c / \omega_{p e}=0.3$ to 30 ). There is no agreement between the observations and a model with quasi-parallel Alfvén waves (Fig. 7h).

We conclude that the observations of the magnetic PSD by STAFF-SA are consistent with permanent fluctuations with a vanishing rest frame frequency but Doppler-shifted up to $f_{l h}$ and $f_{c e}$ in the spacecraft frame. The distribution of the wave vectors of these fluctuations has to be strongly anisotropic, with a spectral density depending on $\theta_{k B}$, like $\left|\sin \theta_{k B}\right|^{100}$ and on $k$, like $k^{-v}$, with $v=3$ to 4 (see Fig. 8a). The wave vector range is $k c / \omega_{p e}=0.3$ to 30 . These permanent fluctuations with a vanishing rest frame frequency and with wave vectors mostly perpendicular to $\boldsymbol{B}$ could be mirror or slow fluctuations. According to Génot et al. (2001), the polarisation of the magnetic and electric fluctuations of purely growing modes, like the mirror modes, is always linear. The polarisation of the observed magnetic fluctuations (see Figs. $2 b$ and $2 \mathrm{~d}$ ) and the polarisation of the observed electric fluctuations (not shown) are mainly linear in the whole e.m. frequency range. This is another argument in favour of purely growing linear modes like the mirror mode. Arguments against the presence of linear mirror modes or slow modes, based on a comparison of the intensity of the electric and of the magnetic fluctuations, will be given in Sect. 7.1.

In this section, we have used analytical approximations of the dispersion relation of kinetic linear wave modes in a 

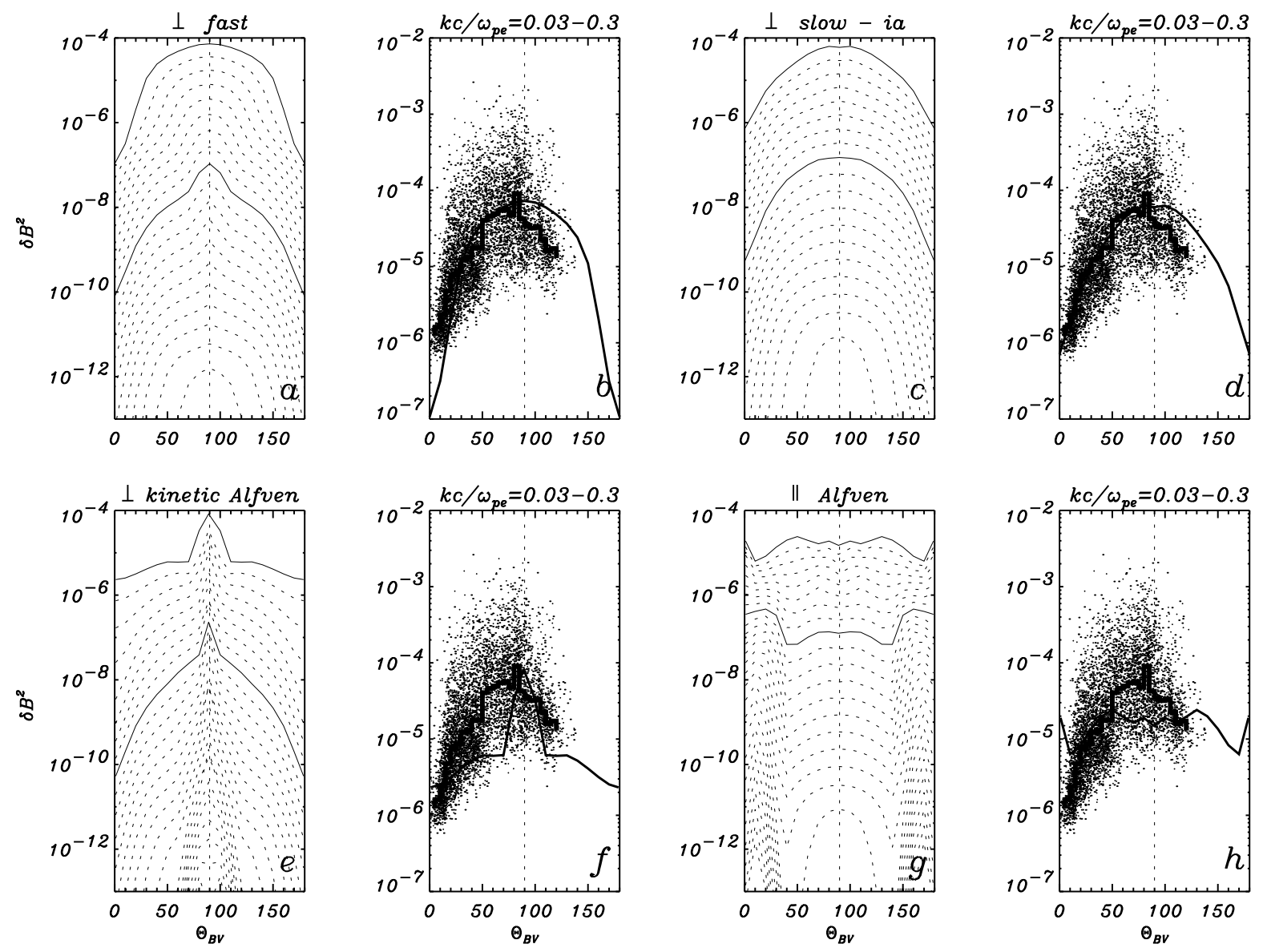

Fig. 7. The model $\delta B^{2}\left(f, \Theta_{B V}\right)$ (Eq. 4), parameters and observations on 19 December 2001. For $k c / \omega_{p e} \simeq 0.3$ to 30 , the fluctuations suffer a pure Doppler shift, with $I_{3 D}(\boldsymbol{k})$ given by Eqs. (5) and (7), $v=3$ to 4 and $\mu=100$. For larger scales $\left(k c / \omega_{p e} \simeq 0.03\right.$ to 0.3$)$, different (Doppler shifted) dispersion relations and different wave vector distributions are considered. Quasi-perpendicular fast mode (Eqs. 7 and 8 ): (a) the model $\delta B^{2}\left(\Theta_{B V}\right)$ at the STAFF-SA frequencies, from $8.8 \mathrm{~Hz}$ (upper solid line) to $561 \mathrm{~Hz}$ (lower dotted line); arbitrary ordinates; (b) the model at $8.8 \mathrm{~Hz}$ (solid line) compared to the scatter plot and the median of the observations at the same frequency, as a function of $\Theta_{B V}$. Quasi-perpendicular slow-ion acoustic mode (Eqs. 7 and 9): (c) the model $\delta B^{2}\left(\Theta_{B V}\right)$ at different frequencies; (d) the model at $8.8 \mathrm{~Hz}$ compared with the observations. Quasi-perpendicular Alfvén waves in the kinetic range (Eqs. 7 and 11$)$ : (e) the model $\delta B^{2}\left(\Theta_{B V}\right)$ at different frequencies; (f) the model at $8.8 \mathrm{~Hz}$ compared with the observations. Quasi-parallel Alfvén waves (Eqs. 6 and 11): (g) the model $\delta B^{2}\left(\Theta_{B V}\right)$ at different frequencies; (h) the model at $8.8 \mathrm{~Hz}$ compared with the observations.

plasma (Eqs. 8 to 11). The conclusion is that these linear waves, if they are present in our data intervals, must have a vanishing phase velocity and a quasi-perpendicular propagation direction. If we had taken the phase velocities of the nonlinear wave modes given by Stasiewicz (2005), we should have found the same result: a vanishing phase velocity for a quasi-perpendicular propagation.

\section{Electric fluctuations in the "ion acoustic" range}

The electric PSD is maximum for $\Theta_{B V} \simeq 90^{\circ}$ at frequencies below $f_{p i}$ (Sect. 3.2): a model with wave vectors mainly perpendicular to $\boldsymbol{B}$ is thus probably suitable in this e.m. range.
Conversely, at and above $f_{p i}, \delta E^{2}$ is minimum for $\Theta_{B V} \simeq 90^{\circ}$ (Fig. 4): we shall assume that the wave vectors for these small scales are mainly parallel to $\boldsymbol{B}$, according to the results displayed in Fig. 6a . Figure 8b displays our composite model for the $\boldsymbol{k}$ distribution of the electric fluctuations: from $k c / \omega_{p e}=0.04$ to 160 , we assume that $\nu=1,2$ and 4 for increasing $k$ (solid line); the anisotropy varies like $\left|\sin \theta_{k B}\right|^{\mu}$ with $\mu=100$. In the upper range, from $k c / \omega_{p e}=16$ to 160 ( $k \lambda_{D e}=0.1$ to 1$)$, we superimpose a spectrum $\propto k^{-4}, 300$ times more intense, with wave vectors mainly parallel to $\boldsymbol{B}$, like $\left|\cos \theta_{k B}\right|^{\mu}$ (dashed line in Fig. 8b), still with $\mu=100$. 

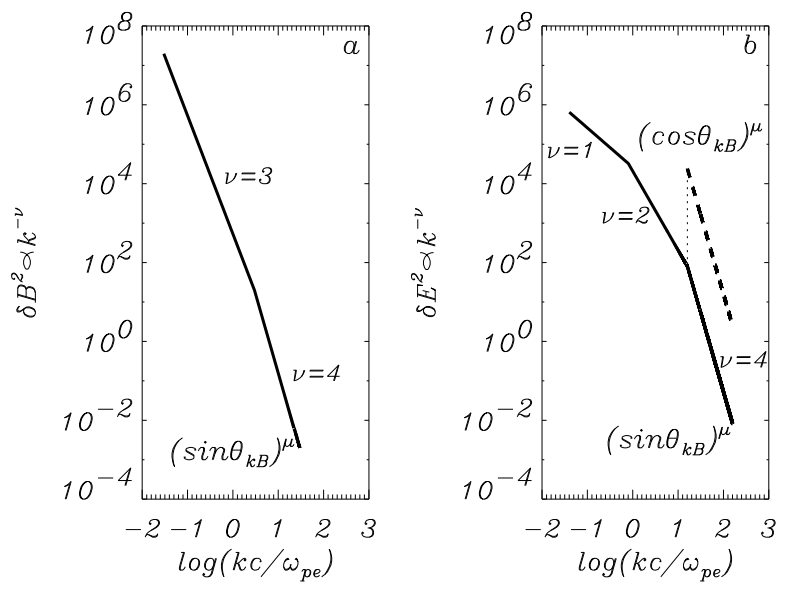

Fig. 8. The models of the wave vector distribution $I_{3 D}(\boldsymbol{k})$ as functions of $k c / \omega_{p e}$ (a) for the magnetic fluctuations $\delta B^{2}$; in our model, the anisotropy with respect to the average $B$ field direction varies like $\left|\sin \theta_{k B}\right|^{\mu}$ with $\mu \simeq 100$; (b) for the electric fluctuations $\delta E^{2}$; in the electromagnetic range (solid line, $k c / \omega_{p e} \simeq 0.03$ to 30 ), the anisotropy model varies like $\left|\sin \theta_{k B}\right|^{\mu}$ with $\mu \simeq 100$. In the electrostatic range (dashed line, $k c / \omega_{p e} \simeq 16$ to 160 ), the anisotropy model varies like $\left|\cos \theta_{k B}\right|^{\mu}$ with $\mu \simeq 100$.

\subsection{Models with pure Doppler effect}

We assume that the composite spectrum of wave vectors (Fig. 8b) simply suffers a Doppler shift. Figure 9a displays the resulting calculated spectra $\delta E^{2}\left(f, \Theta_{B V}\right)$ between $8.8 \mathrm{~Hz}$ (upper dashed line) and $3.6 \mathrm{kHz}$ (lower dashed line). The upper solid line $(11 \mathrm{~Hz})$ is also shown in Fig. 9b superimposed on the observed scatter plot (in $\left.(\mathrm{mV} / \mathrm{m})^{2} / \mathrm{Hz}\right)$ and its median: the observations and the model both display a minimum for $\Theta_{B V} \simeq 0^{\circ}$ and a broad maximum around $\Theta_{B V} \simeq 90^{\circ}$. At higher frequencies (Figs. 9c, 9d and 9e), the global agreement between the model and the data is even better: at $354 \mathrm{~Hz}$ (Fig. 9d) there is a relative minimum of the data for $\Theta_{B V} \simeq 90^{\circ}$. The model (Fig. 9d) varies strongly from $\Theta_{B V}=0^{\circ}$ to $30^{\circ}$ : this is consistent with the large dispersion of the data points at small $\Theta_{B V}$. The spectra of Fig. 9f for large and small angles are similar to the observations of Fig. 4c. If we now suppose that the wave vector distribution of the model is isotropic for $k \lambda \lambda_{D}=0.1$ to 1 , i.e. if $\mu=0$ for the dashed line in Fig. 8b, the spectra (not shown) are the same for large and for small $\Theta_{B V}$ above about $200 \mathrm{~Hz}$ : this is not observed, so that the wave vector distribution of the e.s. fluctuations has to be anisotropic.

\subsection{Models with a nonvanishing rest frame frequency}

Let us now assume that, in the composite $k$ distribution of Fig. 8b, the fluctuations with $\boldsymbol{k}$ perpendicular to $\boldsymbol{B}$ (solid line) still suffer a simple Doppler shift $\left(\omega_{0} \simeq 0\right)$ while the fluctuations with $\boldsymbol{k}$ parallel to $\boldsymbol{B}$ (dashed line) are Doppler-
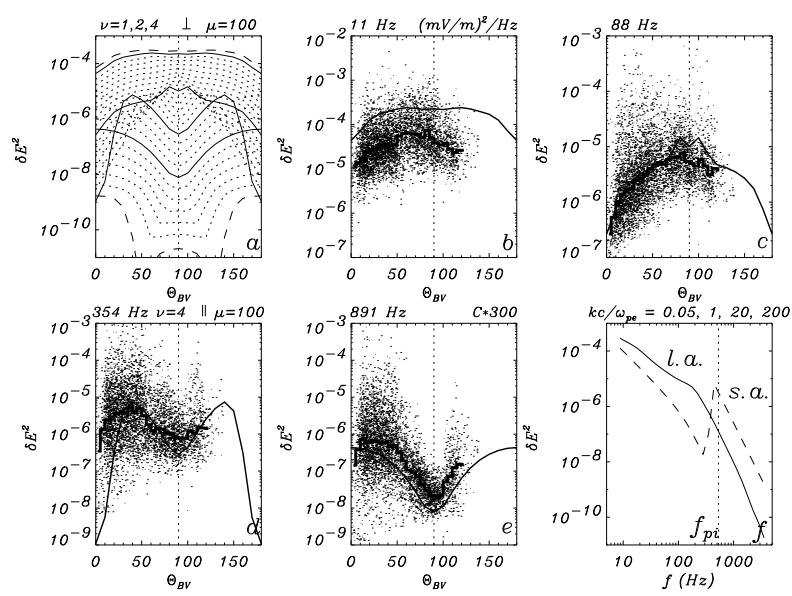

Fig. 9. The model $\delta E^{2}\left(f, \Theta_{B V}\right)$ (Eq. 4) with a simple Doppler shift and no dispersion relation in Eq. (3). The wave vector distribution $I_{3 D}(\boldsymbol{k})$ is given by the composite spectrum of Fig. $8 \mathrm{~b}$. Parameters and observations on 19 December 2001: (a) the model $\delta E^{2}\left(\Theta_{B V}\right)$ at the STAFF-SA frequencies, from $8.8 \mathrm{~Hz}$ (upper dashed line) to $3600 \mathrm{~Hz}$ (lower dashed line). The four solid lines correspond, respectively, to $f=11 \mathrm{~Hz}, 88 \mathrm{~Hz}, 354 \mathrm{~Hz}$ and $891 \mathrm{~Hz}$; arbitrary ordinates; (b) the model at $11 \mathrm{~Hz}$ (solid line) compared to the scatter plot and the median of the observations at the same frequency, as a function of $\Theta_{B V}$; (c) the model and the observations at $88 \mathrm{~Hz},(\mathbf{d})$ at $354 \mathrm{~Hz},(\mathbf{e})$ at $891 \mathrm{~Hz}$; (f) the model spectra for large $\Theta_{B V}$ angles (solid line, l.a.) and for small $\Theta_{B V}$ angles (dashed line, s.a.). The vertical dotted line gives the average value of $f_{p i}$ on 19 December 2001.

shifted ion acoustic waves, with a frequency $\omega_{0}$ given by Eq. (9) $\left(\omega_{0} \simeq k c_{s} \cos \theta_{k B}\right)$ with $c_{s} \simeq 150 \mathrm{~km} / \mathrm{s}$. We see that the calculated spectra (with ion acoustic waves) given in Figs. 10b, 10c, 10d and e are consistent with the observations, as were the calculated spectra (without ion acoustic waves) of Figs. 9b, 9c, 9d and 9e. Above $500 \mathrm{~Hz}$ (Fig. 10f) the model spectrum for large $\Theta_{B V}$ is about 10 times weaker than the spectrum for small $\Theta_{B V}$; this is observed in Fig. 4c.

If we now suppose that the model of the wave vector distribution of the ion acoustic waves is isotropic, i.e. if $\mu=0$ for the dashed line in Fig. 8b, the 1.a. and s.a. spectra (not shown) are not the same above about $500 \mathrm{~Hz}$ : the 1.a. spectrum is 2 to 3 times weaker than the s.a. spectrum. Indeed, the ion acoustic phase velocity vanishes at large $\theta_{k B}$, so that large (less intense) $k$ is needed to reach the same frequency when $\Theta_{B V}$ is large. As we observe that the 1.a. spectrum is at least 10 times weaker than the s.a. spectrum (Fig. 4c), the wave vector distribution of the ion acoustic waves has to be anisotropic. But the presence of ion acoustic waves, with a phase velocity $\propto \cos \theta_{k B}$, amplifies the part played by the anisotropic wave vector distribution, peaking for $\boldsymbol{k}$ parallel to $\boldsymbol{B}$, more especially as the sound speed is close to the flow speed. 

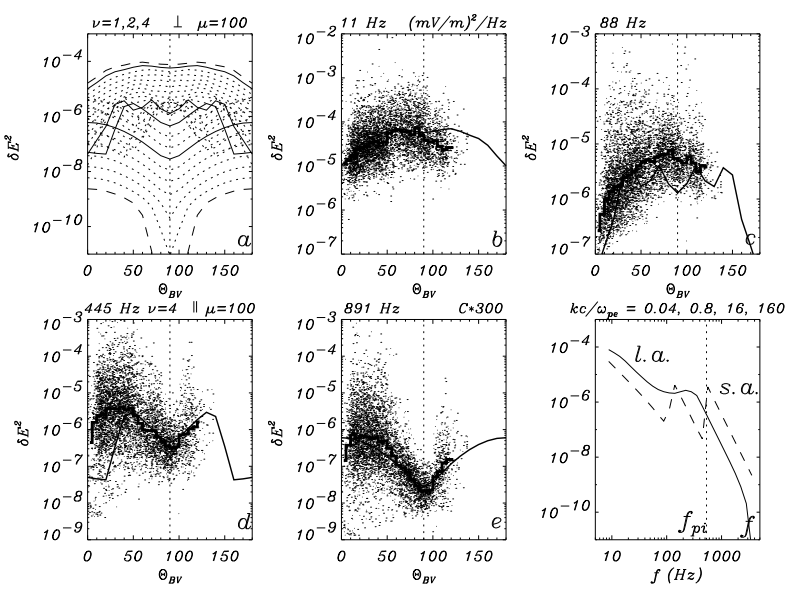

Fig. 10. The model $\delta E^{2}\left(f, \Theta_{B V}\right)$ (Eq. 4) for the composite spectrum $I_{3 D}(\boldsymbol{k})$ of Fig. $8 \mathrm{~b}$ : the electromagnetic fluctuations corresponding to the solid line in Fig. 8b suffer a simple Doppler shift; the electrostatic fluctuations corresponding to the dashed line in Fig. 8b suffer a Doppler shift and have the dispersion relation of the slow-ion acoustic mode (Eq. 9): (a) the model $\delta E^{2}\left(\Theta_{B V}\right)$ at the STAFF-SA frequencies (see the caption of Fig. 9a). The four solid lines correspond, respectively, to $f=11 \mathrm{~Hz}, 88 \mathrm{~Hz}, 445 \mathrm{~Hz}$ and $891 \mathrm{~Hz}$; arbitrary ordinates; (b) the model at $11 \mathrm{~Hz}$ (solid line) (see the caption of Fig. 9b); (c) the model and the observations at $88 \mathrm{~Hz}$, (d) at $445 \mathrm{~Hz}$, (e) at $891 \mathrm{~Hz}$; (f) the model spectra for large and small $\Theta_{B V}$ (see the caption of Fig. 9f).

We conclude that the permanent electric (e.m.) fluctuations $\delta E^{2}$ observed between $f_{l h}$ and $f_{c e} \simeq f_{p i}$ have a vanishing rest frame frequency and are Doppler-shifted up to $f_{l h}$ and $f_{c e}$; the distribution function of their wave vectors peaks like $\left|\sin \theta_{k B}\right|^{100}$ i.e. $\boldsymbol{k}$ perpendicular to $\boldsymbol{B}$, and varies like $k^{-v}$, with $\nu=1$ to 2 ; the wave vector range is $k c / \omega_{p e} \simeq 0.05$ to 20. At smaller scales, $k c / \omega_{p e} \simeq 20$ to 200 , the electric (e.s.) fluctuations may have a vanishing frequency $\omega_{0} \simeq 0$, but the observations are also statistically consistent with Doppler shifted ion acoustic waves; the distribution of the wave vectors has to peak like $\left|\cos \theta_{k B}\right|^{100}$, i.e. $\boldsymbol{k}$ parallel to $\boldsymbol{B}$, with a power law dependence $\propto k^{-4}$.

\section{Discussion}

\subsection{Wave modes in the e.m. range?}

In Sect. 5.2, we have seen that the magnetic fluctuations in the e.m. range have a vanishing frequency. The electric fluctuations in the same frequency range also have a vanishing frequency (Sect. 6.1). If interpreted with a linear theory, these e.m. fluctuations could thus be mirror structures or slow mode structures with wave vectors quasi-perpendicular to $\boldsymbol{B}$.

To try to identify the wave mode of the permanent e.m. fluctuations, we consider the ratio $\delta E^{2} / \delta B^{2}$ observed at the e.m. frequencies. On 19 December 2001, the observed ratio $\delta E^{2} / \delta B^{2}$ averaged over $6 \mathrm{~h}$ (in $\left.(\mathrm{mV} / \mathrm{m})^{2} / \mathrm{nT}^{2}\right)$ varies from about 10 at $10 \mathrm{~Hz}$ to $10^{5}$ at $400 \mathrm{~Hz}$ in the plasma frame (from the observed values of $\left.\sqrt{(} \delta E^{2}\right)$ in the spacecraft frame, we have substracted the electric field $\left(V \sqrt{(} \delta B^{2}\right)$ which is an upper value for the induced electric field $(V \times \delta B)$, and which is negligible). Using the program WHAMP, we calculate the ratio $\delta E^{2} / \delta B^{2}$ for the different kinetic linear modes at $\theta_{k B}=85^{\circ}$ and for $10^{-2}<k c / \omega_{p e}<0.3 ; \delta E^{2} / \delta B^{2}$ is $10^{-5}$ to $10^{-3}$ for the mirror mode, $310^{-3}$ to 1 for the slow mode, 3 $10^{-2}$ for the Alfvén mode, and $510^{-2}$ to 3 for the fast mode: there is no linear quasi-perpendicular mode with $\delta E^{2} / \delta B^{2}$ as large as the observed values. (The slow mode for a quasiparallel propagation is the only mode for which $\delta E^{2} / \delta B^{2}$ reaches 10 to $10^{5}$ ). However, the linear kinetic model of WHAMP at large scales cannot be used for a mode identification at smaller scales, especially as the observed small-scale fluctuations can be in a highly nonlinear state.

\subsection{Symmetries with respect to $\Theta_{B V}=90^{\circ}$}

When the angle $\Theta_{B V}$ varies from $0^{\circ}$ to $180^{\circ}$, the scatter plot $\delta B^{2}\left(\Theta_{B V}\right)$ around $f_{l h}$ is symmetrical with respect to $\Theta_{B V}=90^{\circ}$ (17 May 2002, Fig. 1c); but $\delta E^{2}\left(\Theta_{B V}\right)$ around $f_{p i}$ is not symmetrical (Fig. 5f): it is 10 times larger for $\Theta_{B V}=45^{\circ}$ than for $\Theta_{B V}=135^{\circ}$.

It is well known that the wave vectors of Alfvén waves in the magnetosheath are generally directed downstream, so that $k_{x} \equiv \boldsymbol{k} . \boldsymbol{V}>0$ (Matsuoka et al., 2000). Can the asymmetry of Fig. $5 \mathrm{f}$ be due to an asymmetry of the distribution of $k_{x}$ for e.s. fluctuations with a nonvanishing $\omega_{0}$ ?

At a given frequency $\omega$, the solutions $k_{x s}$ of Eq. (1) are the same for $\Theta_{B V}=45^{\circ}$ and for $\Theta_{B V}=135^{\circ}$, because we assume that waves with $\boldsymbol{k} . \boldsymbol{B}>0$ and waves with $\boldsymbol{k} . \boldsymbol{B}<0$ have the same positive frequency $\omega_{0}$. The waves with $\boldsymbol{k} . \boldsymbol{B}>0$ for $\Theta_{B V}=45^{\circ}$ will suffer the same positive Doppler shift $\left(k_{x s}>0\right)$ as the waves with $\boldsymbol{k} \cdot \boldsymbol{B}<0$ for $\Theta_{B V}=135^{\circ}$. Thus, if we withdraw the solutions $k_{x s}<0$ in the integral (Eq. 4), we simply obtain $\delta E^{2}\left(\Theta_{B V}\right)$ two times weaker than if we consider both $k_{x s}<0$ and $k_{x s}>0: \delta E^{2}\left(f, \Theta_{B V}\right)$ remains symmetrical with respect to $\Theta_{B V}=90^{\circ}$.

The only way to obtain asymmetries in $\delta E^{2}\left(\Theta_{B V}\right)$ would be to have waves propagating with only $\boldsymbol{k} . \boldsymbol{B}>0$, because the Doppler shift would increase $\omega$ for $\Theta_{B V}=45^{\circ}\left(k_{x s}>0\right)$, and would decrease $\omega$ for $\Theta_{B V}=135^{\circ}\left(k_{x s}<0\right)$. But waves with only $\boldsymbol{k} . \boldsymbol{B}>0$ (or only $\boldsymbol{k} . \boldsymbol{B}<0$ ) are not observed.

Figure 11 shows that the asymmetry observed on 17 May 2002 (Fig. 5f) is probably due to the fact that the plasma properties were different for $\Theta_{B V}<90^{\circ}$ and for $\Theta_{B V}>90^{\circ}$ : the plasma density (Fig. 11b) and the proton temperature (Fig. 11d) were higher for $\Theta_{B V}>90^{\circ}$, the magnetic field was weaker (Fig. 11c); the flow speed (Fig. 11a) and the electron temperature (not shown) were nearly the same. 

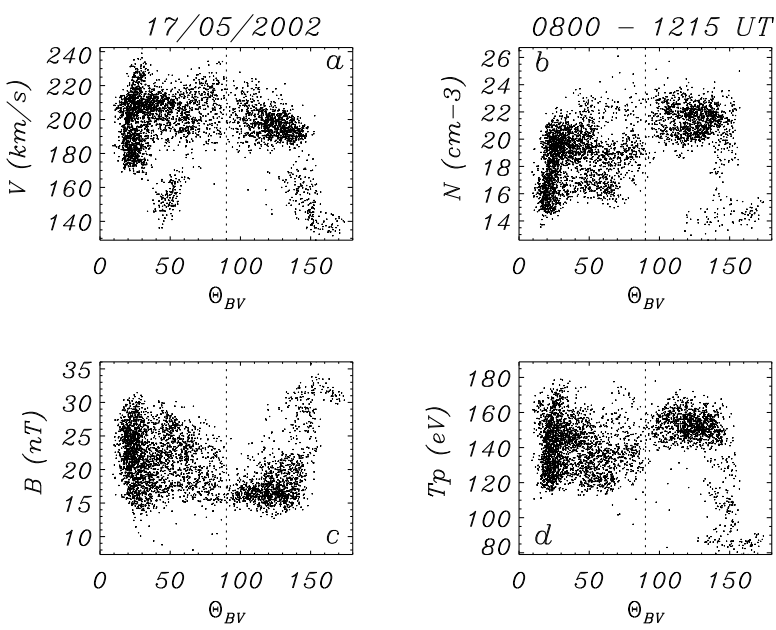

Fig. 11. 17 May 2002, observations between 08:00 and 12:15 UT, as functions of the angle $\Theta_{B V}$, (a) the flow speed, (b) the proton density, (c) the $B$ field intensity, (d) the proton temperature.

\subsection{Comparison with the solar wind turbulence}

The magnetosheath is made of the solar wind plasma compressed through the Earth's bow shock. There are similarities between the anisotropies of the wave vector distributions in the solar wind and in the magnetosheath.

In the electrostatic range ( $f \simeq 4-6 \mathrm{kHz}, k \lambda_{D e} \simeq 0.3$ ) the intensity $\delta E^{2}$ of the electric fluctuations in the solar wind is minimum when the angle $\Theta_{B X}$ between $\boldsymbol{B}$ and the GSE $\boldsymbol{X}$-axis is about $90^{\circ}$ (Lacombe et al., 2002). As $\Theta_{B X} \simeq \Theta_{B V}$ in the solar wind, this minimum can be due to the Doppler shift: in the solar wind, as well as in the magnetosheath, the e.s. wave vectors are mostly parallel to $\boldsymbol{B}$ in the range $k \lambda \lambda_{D e} \geq 0.1$.

In the MHD range $\left(f \simeq 10^{-2} \mathrm{~Hz}\right)$, i.e. in the inertial range of the electromagnetic solar wind turbulence, the total intensity $\delta B^{2}$ in the three directions depends on $\Theta_{B V}$ : Bieber et al. (1996) observe that $\delta B^{2}$ increases when $\Theta_{B V}$ increases up to $90^{\circ}$ in the solar wind. These observations imply that the wave vectors are mostly perpendicular to $\boldsymbol{B}$. As for the wave modes, Bale et al. (2005) suggest that kinetic Alfvén waves could account for their observations of the electric field in the solar wind, in the inertial and proton dissipation ranges.

In the magnetosheath, at electron scales $k c / \omega_{p e} \simeq 0.3$ to 30 , we find that the wave vectors of the permanent magnetic fluctuations are mainly perpendicular to $\boldsymbol{B}$, as in the solar wind at larger scales; but the variance of the magnetic fluctuations is nearly isotropic in the magnetosheath, while this variance is minimum along $\boldsymbol{B}$ in the solar wind. At the same electron scales, the electric fluctuations also have wave vectors mainly perpendicular to $\boldsymbol{B}$, but no dispersion relation is consistent with the observations of $\delta B^{2}$ and $\delta E^{2}$ as functions of $f$ and of the angle $\Theta_{B V}$.
Sahraoui et al. (2004) analyse an interval of magnetic fluctuations in the magnetosheath, up to $1.4 \mathrm{~Hz}$, in a high$\beta$ plasma near the magnetopause. They observe a mirror mode, Doppler-shifted at $0.11 \mathrm{~Hz}$, which corresponds to the largest linear growth rate for the observed plasma parameters; they also observe mirror modes Doppler-shifted up to $1.4 \mathrm{~Hz}$, as a nonlinear extension of the most unstable mode to smaller scales; the wave vectors are mostly perpendicular to $\boldsymbol{B}$. During our interval, 19 December 2001, according to WHAMP, the quasi-perpendicular mirror modes are not unstable, while the quasi-parallel AIC waves are linearly unstable (see Sect. 5.2). However, the observations of $\delta B^{2}\left(f, \Theta_{B V}\right)$ are consistent with quasi-perpendicular wave vectors in the e.m. range $k c / \omega_{p e} \simeq 0.3$ to 30 . We thus find that the wave vectors of the permanent fluctuations in the magnetosheath are mostly perpendicular to $\boldsymbol{B}$ at electron scales, in the range $k c / \omega_{p e} \simeq 0.3$ to 30 , even if the unstable dominant modes at larger scales are not quasi-perpendicular mirror modes but are quasi-parallel AIC waves.

Carbone et al. (1995) consider the magnetic fluctuations below $1 \mathrm{~Hz}$ during Alfvénic periods in the solar wind. They analyse separately the Alfvénic (A) polarisation and the compressive (S) polarisation. For the A polarisation, $\boldsymbol{k}$ is observed to be mainly parallel to $\boldsymbol{B}$; for the $S$ polarisation, the $\boldsymbol{k}$ distribution is flattened in the $(\boldsymbol{B}, \boldsymbol{V})$ plane. This last result implies that the $\boldsymbol{k}$ distributions are not axisymmetric with respect to $\boldsymbol{B}$. In the magnetosheath near the magnetopause, Sahraoui et al. (2006) also find a $\boldsymbol{k}$ distribution which is not axisymmetric.

At MHD scales, the cascade from small to large wave numbers is different in the directions parallel and perpendicular to the $\boldsymbol{B}$ field (see the review by Oughton and Matthaeus, 2005): the parallel cascade is likely to be rather weak in the solar wind. This difference is probably still present at the electron MHD scales $\left(k c / \omega_{p e} \simeq 1\right)$ in the magnetosheath: we find wave vectors mostly perpendicular to $\boldsymbol{B}$ for the e.m. fluctuations.

In our observations, we have only considered the trace $\delta B^{2}$ of the magnetic fluctuation tensor, and we have assumed in our model that the $\boldsymbol{k}$ distributions were axisymmetric at the electron scales. In a future work, we shall check whether non-axisymmetric $\boldsymbol{k}$ distributions in the magnetosheath would be consistent with the slightly anisotropic distribution of the variance of the e.m. fluctuations observed around $10 \mathrm{~Hz}$.

\subsection{Energy dissipation?}

In the STAFF-SA frequency range, the magnetic and electric fluctuations are more intense in the magnetosheath than in the solar wind and in the magnetosphere. This could be due to a continuous dissipation of part of the solar wind energy: such a dissipation indeed begins at the bow shock.

The solar wind Poynting vector $\boldsymbol{S}_{S W} \propto \boldsymbol{E}_{S W} \times \boldsymbol{B}_{S W}$ gives the large-scale e.m. energy flux which impinges on the mag- 
netosphere obstacle. As $\boldsymbol{E}_{S W}=-\boldsymbol{V}_{S W} \times \boldsymbol{B}_{S W}, \boldsymbol{S}_{S W}$ varies like $V_{S W} B_{S W}^{2} \sin \theta_{B V}^{S W}$. We have seen that the PSD $\delta B^{2}$ increases like the local $\sin \Theta_{B V}$ in the magnetosheath. Is this increase due to a dissipation of $\boldsymbol{S}_{S W}$ ? Let us first assume that the local quantities in the magnetosheath $V, B, \sin \Theta_{B V}$ (and $S \propto V B^{2} \sin \Theta_{B V}$ ) are proportional to the solar wind quantities $V_{S W}, B_{S W}, \sin \theta_{B V}^{S W}$ (and $S_{S W}$ ). If $\delta B^{2}$ was proportional to $S$, it would increase when $\sin \Theta_{B V}$ increases (which is observed) but also when $B^{2}$ increases, and this is not observed: on the four days considered, $\delta B^{2}$ is constant or decreases when $B^{2}$ increases, so that $\delta B^{2}$ is not proportional to $S$. Furthermore, the numerical simulations of Paper 2 show that even if the magnetosheath intensities of $V$ and $B$ are related to the solar wind intensities of $V_{S W}$ and $B_{S W}$, there is no simple relation between $\theta_{B V}^{S W}$ and $\Theta_{B V}$ in the magnetosheath: indeed, for a given $\theta_{B V}^{S W}, \Theta_{B V}$ strongly depends on the position in the magnetosheath, so that $S$ is not proportional to $S_{S W}$. The increase in $\delta B^{2}$ with the angle $\Theta_{B V}$ is thus probably not due to the dissipation of the large-scale solar wind Poynting vector in the magnetosphere frame, it is mainly due to the Doppler shift.

Similarly, we have checked that the increase in $\delta B^{2}$ with $\Theta_{B V}$ in the e.m. range was not due to the clock angle of the solar wind magnetic field $B_{S W}$, or to local velocity shears. As for the dependences of $\delta B^{2}$ and $\delta E^{2}$ on the solar wind dynamical pressure and on the cone angle of $B_{S W}$, they will be addressed in Paper 2.

\section{Conclusions}

In this paper, we have not considered the fluctuations like the e.m. whistler or the e.s. solitary waves which are sometimes observed in the magnetosheath, but only the underlying permanent fluctuations.

At a given frequency in the magnetosheath, the permanent electromagnetic Power Spectral Densities $\delta B^{2}$ and $\delta E^{2}$ (below about $300 \mathrm{~Hz}$ in the spacecraft frame) strongly depend on the local angle $\Theta_{B V}$ between the magnetic field $\boldsymbol{B}$ and the flow velocity $V: \delta B^{2}$ and $\delta E^{2}$ peak when $\Theta_{B V}$ is around $90^{\circ}$. This is due to the Doppler shift of the fluctuations, the frequency of which is $f_{0} \simeq 0$ in the plasma frame. This implies that at the electron scales ranging from $k c / \omega_{p e} \simeq 0.3$ to 30 , the distribution of the wave vectors is strongly peaked for $\boldsymbol{k}$ perpendicular to $\boldsymbol{B}$, like $\left|\sin \theta_{k B}\right|^{\mu}$, with $\mu \simeq 100$, and increases at large scales, like $k^{-\nu}$. In this pure Doppler model, the slope $v$ of the 1-D wave vector spectrum is equal to the slope $v \simeq 3$ to 4 of the frequency spectrum in the spacecraft frame. We emphasize that the wave vectors of the permanent e.m. fluctuations at electron scales are perpendicular to $\boldsymbol{B}$, regardless of the wave vectors' direction for the unstable waves at larger scales (quasi-parallel for Alfvén ion cyclotron waves, quasi-perpendicular for mirror modes).

The ratio $\delta E^{2} / \delta B^{2}$ observed in the e.m. range is much larger than this ratio calculated for the linear kinetic wave modes, for a quasi-perpendicular propagation $\theta_{k B}=85^{\circ}$ and for $10^{-2}<k c / \omega_{p e}<0.3$. We conclude that there is no indication of the presence of linear wave modes in this range: the electric and the magnetic fluctuations do not belong to a same linear wave mode. Nevertheless, there is a crude correlation between the time variations of $\delta E^{2}$ and $\delta B^{2}$ at a given frequency because these two kinds of fluctuations have similar $\boldsymbol{k}$ distributions and thus suffer the same Doppler shift at the same time.

At smaller scales $k c / \omega_{p e} \simeq 20$ to 200 (i.e. $k \lambda_{D e} \simeq 0.15$ to 1.5), the variations of $\delta E^{2}$ with $\Theta_{B V}$ imply that the distribution of the wave vectors of the electrostatic fluctuations is peaked for $\boldsymbol{k}$ parallel to $\boldsymbol{B}$, like $\left|\cos \theta_{k B}\right|^{\mu}$, with $\mu \simeq 100$. The observations $\delta E^{2}\left(\Theta_{B V}\right)$ are consistent with $f_{0}=0$ in the plasma frame; they are also consistent with the presence of quasi-parallel dispersive ion acoustic waves in the electron dissipation range (related to the Debye length). To account for the observed variations of $\delta E^{2}$ with the frequency and with $\Theta_{B V}$, the electrostatic fluctuations $\delta E_{e s}^{2}$ which peak like $\left|\cos \theta_{k B}\right|^{100}$ have to be about 300 times more intense, at $k c / \omega_{p e} \simeq 30$, than the electromagnetic fluctuations $\delta E_{e m}^{2}$ which peak like $\left|\sin \theta_{k B}\right|^{100}$.

The distributions of the magnetosheath wave vectors $\boldsymbol{k}$, shown in Figs. 8a and 8b, have not been obtained by a deconvolution of the data but by a fitting with a model implying several parameters. This model is not unique and we cannot really state that more complex models with dispersive waves (and, for example, an anisotropy index $\mu$ depending on $k$ ) are excluded by the observations. However, the fit between the relatively simple axisymmetric models described here and the data is surprisingly good. This means that, in the magnetosheath, some properties of the magnetic and electric turbulence, at scales smaller than the spacecraft separation, can be determined thanks to the Doppler effect, and thanks to the good coverage of the range $0^{\circ}-90^{\circ}$ by the angle $\Theta_{B V}$.

As mentioned in the Introduction, the Taylor hypothesis implies that the wave frequency $f_{0}$ in the plasma rest frame is vanishing, i.e., that the phase speed of the wave $2 \pi f_{0} / k$ is much smaller than the flow speed $V$. This hypothesis is usually made in the solar wind, in the MHD range and in the dissipation range. In the Earth's magnetosheath, the flow speed is smaller than the solar wind speed, while the characteristic frequencies, $f_{c i}$ and $f_{p i}$, are slightly larger than in the solar wind. The Taylor hypothesis $2 \pi f_{0} / k \ll V$ could thus be less valid in the magnetosheath: any dispersion effect occurring in the dissipation range could be more evident in the magnetosheath than in the solar wind. However, we have shown that in the electron MHD (electromagnetic) range $k c / \omega_{p e} \simeq 0.3$ to 30 , the Taylor hypothesis is valid for the permanent fluctuations in the magnetosheath: $f_{0}$ is vanishing in the plasma rest frame, and there is no indication of statistically important fast waves, Alfvén waves or slow waves (meanwhile, the linear polarisation of the magnetic and electric fluctuations is consistent with the permanent presence of purely growing modes in the electron MHD range, see Sect. 7.1). 
In the electrostatic range, the presence of ion acoustic waves is possible. The sound speed is weaker than the flow speed, but it is not negligible: the Taylor hypothesis is generally less valid than in the e.m. range.

We emphasize that the electric or magnetic PSD, at a given frequency below about $3 \mathrm{kHz}$ in the spacecraft frame, can be multiplied by 10 to $10^{3}$ when the angle $\Theta_{B V}$ varies. We thus have to take into account this consequence of the Doppler effect if we want to study the other parameters which control the intensity of the magnetic and electric fluctuations in the magnetosheath. This will be done in Paper 2.

Acknowledgements. We are very grateful to the team of the Cluster Magnetic field investigation (PIs A. Balogh and E. Lucek). We thank A. Fazakerley for the use of the Peace data. It is a pleasure to thank J. Pickett for useful discussions, and O. Santolìk for the program PRASSADCO which gives the polarisation of the fluctuations in the frequency range of STAFF-SA. We thank the three referees for useful comments.

Topical Editor I. A. Daglis thanks three referees for their help in evaluating this paper.

\section{References}

Alexandrova, O., Mangeney, A., Maksimovic, M., Lacombe, C., Cornilleau-Wehrlin, N., Lucek, E. A., Décréau, P. M. E., Bosqued, J.-M., Trávníček, P., and Fazakerley, A. N.: Cluster observations of finite amplitude Alfvén waves and small-scale magnetic filaments downstream of a quasi-perpendicular shock, J. Geophys. Res., 109, A05207, doi:10.1029/2003JA010056, 2004.

Bale, S. D., Kellogg, P. J., Mozer, F. S., Horbury, T. S., and Rème, H.: Measurements of the electric fluctuation spectrum of magnetohydrodynamic turbulence, Phys. Rev. Letters, 94, 215002, doi:10.1103/PhysRevLett.94.215002, 2005.

Balogh, A., Dunlop, M. W., Cowley, S. W. H., Southwood, D. J., Thomlinson, J. G., Glassmeier, K. H., Musmann, G., Lühr, H., Buchert, S., Acuña, M. H., Fairfield, D. H., Slavin, J. A., Riedler, W., Schwingenschuh, K., Kivelson, M. G., and the Cluster magnetometer team: The Cluster Magnetic Field Investigation, Space Science Reviews, 79, 65-92, 1997.

Baumjohann, W. and Treumann, R. A.: Basic Space Plasma Physics, Imperial College Press, 1996.

Bieber, J. W., Wanner, W., and Matthaeus, W. H.: Dominant twodimensional solar wind turbulence with implications for cosmic ray transport, J. Geophys. Res., 101, 2511-2522, 1996.

Carbone, V., Malara, F., and Veltri, P.: A model for the threedimensional magnetic field correlation spectra of low-frequency solar wind fluctuations during Alfvénic periods, J. Geophys. Res., 100, 1763-1778, 1995.

Cornilleau-Wehrlin, N., Chauveau, P., Louis, S., Meyer, A., Nappa, J. M., Perraut, S., Rezeau, L., Robert, P., Roux, A., de Villedary, C., de Conchy, Y., Friel, L., Harvey, C. C., Hubert, D., Lacombe, C., Manning, R., Wouters, F., Lefeuvre, F., Parrot, M., Pinçon, J.L., Poirier, B., Kofman, W., Louarn, P., and the Staff investigator team: The Cluster Spatio-temporal Analysis of Field Fluctuations (Staff) Experiment, Space Science Reviews, 79, 107-136, 1997.
Cornilleau-Wehrlin, N., Chanteur, G., Perraut, S., Rezeau, L., Robert, P., Roux, A., de Villedary, C., Canu, P., Maksimovic, M., de Conchy, Y., Hubert, D., Lacombe, C., Lefeuvre, F., Parrot, M., Pinçon, J.-L., Décréau, P. M. E., Harvey, C. C., Louarn, P., Santolìk, O., Alleyne, H. St. C., Roth, M., Chust, T., Le Contel, O., and Staff team: First results obtained by the Cluster STAFF Experiment, Ann. Geophys., 21, 437-456, 2003, http://www.ann-geophys.net/21/437/2003/.

Coroniti, F. V., Greenstadt, E. W., Moses, S. L., Tsurutani, B. T., and Smith, E. J.: On the absence of plasma wave emissions and the magnetic field orientation in the distant magnetosheath, Geophys. Res. Lett, 21, 2761-2764, 1994.

Génot, V., Schwartz, S. J., Mazelle, C., Balikhin, M., Dunlop, M., and Bauer, T. M.: Kinetic study of the mirror mode, J. Geophys. Res., 106, 21 611-21 622, 2001.

Horbury, T. S., Lucek, E. A., Balogh, A., Dandouras, I., and Rème, H.: Motion and orientation of magnetic field dips and peaks in the terrestrial magnetosheath, J. Geophys. Res., 109, A09209, doi:10.1029/2003JA010237, 2004.

Hubert, D., Lacombe, C., Harvey, C. C., Moncuquet, M., Russell, C. T., and Thomsen, M. F.: Nature, properties, and origin of low-frequency waves from an oblique shock to the inner magnetosheath, J. Geophys. Res., 103, 26 783-26 798, 1998.

Johnstone, A. D., Alsop, C., Burge, S., Carter, P. J., Coates, A. J., Coker, A. J., Fazakerley, A. N., Grande, M. Gowen, R. A., Gurgiolo, C., Hancock, B. K., Narheim, B., Preece, A., Sheather, P. H., Winningham, J. D., and Woodliffe, R. D.: Peace: a Plasma Electron and Current Experiment, Space Science Reviews, 79, 351-398, 1997.

Lacombe, C. and Belmont, G.: Waves in the Earth's magnetosheath: observations and interpretations, Adv. Space Res., 15(8/9), 329340, 1995 .

Lacombe, C., Belmont, G., Hubert, D., Harvey, C. C., Mangeney, A., Russell, C. T., Gosling, J. T., and Fuselier, S. A.: Density and magnetic field fluctuations observed by ISEE 1-2 in the quiet magnetosheath, Ann. Geophys., 13, 343-357, 1995, http://www.ann-geophys.net/13/343/1995/.

Lacombe, C., Salem, C., Mangeney, A., Hubert, D., Perche, C., Bougeret, J.-L., Kellogg, P. J., and Bosqued, J.-M.: Evidence for the interplanetary electric potential? WIND observations of electrostatic fluctuations, Ann. Geophys., 20, 609-618, 2002, http://www.ann-geophys.net/20/609/2002/.

Lacombe, C., Samsonov, A. A., Mangeney, A., Maksimovic, M., and Trávníček, P.: Cluster observations in the magnetosheath Part 2: Intensity of the turbulence at electron scales, Ann. Geophys., 24, 3523-3531, 2006, http://www.ann-geophys.net/24/3523/2006/.

Lucek, E. A., Dunlop, M. W., Horbury, T. S., Balogh, A., Brown, P., Cargill, P., Carr, C., Fornaçon, K.-H., Georgescu, E., and Oddy, T.: Cluster magnetic field observations in the magnetosheath: four-point measurements of mirror structures, Ann. Geophys., 19, 1421-1428, 2001, http://www.ann-geophys.net/19/1421/2001/.

Lucek, E. A., Constantinescu, D., Goldstein, M. L., Pickett, J. S., Pinçon, J.-L., Sahraoui, F., Treumann, R. A., and Walker, S. N.: The Magnetosheath, Space Science Reviews, 118, 95-152, 2005.

Matsuoka, A., Southwood, D. J., Kokubun, S., and Mukai, T.: Propagation sense of low-frequency MHD waves in the magnetosheath observed by Geotail, J. Geophys. Res., 105, 18361- 
$18376,2000$.

Oughton, S. and Matthaeus, W. H.: Parallel and perpendicular cascades in solar wind turbulence, Nonlin. Processes Geophys., 12, 299-310, 2005, http://www.nonlin-processes-geophys.net/12/299/2005/.

Pickett, J. S., Menietti, J. D., Gurnett, D. A., Tsurutani, B., Kintner, P. M., Klatt, E., and Balogh, A.: Solitary potential structures observed in the magnetosheath by the Cluster spacecraft, Nonlin. Processes Geophys., 10, 3-11, 2003,

http://www.nonlin-processes-geophys.net/10/3/2003/.

Pickett, J. S., Chen, L.-J., Kahler, S. W., Santolik, O., Goldstein, M. L., Lavraud, B., Décréau, P. M. E., Kessel, R., Lucek, E., Lakhina, G. S., Tsurutani, B. T., Gurnett, D. A., CornilleauWehrlin, N., Fazakerley, A., Rème, H., and Balogh, A.: On the generation of solitary waves observed by Cluster in the nearEarth magnetosheath, Nonlin. Processes Geophys., 12, 181-193, 2005 , http://www.nonlin-processes-geophys.net/12/181/2005/.

Rème, H., Bosqued, J.-M., Sauvaud, J. A., Cros, A., Dandouras, J., Aoustin, C., Bouyssou, J., Camus, T., Cuvilo, J., Martz, C., Médale, J. L., Perrier, H., Romefort, D., Rouzaud, J., d'Uston, C., Möbius, E., Crocker, K., Granoff, M., Kistler, L. M., Popecki, M., Hovestadt, D., Klecker, B., Paschmann, G., Scholer, M., Carlson, C. W., Curtis, D. W., Lin, R. P., McFadden, J. P., Formisano, V., Amata, E., Bavassano-Cattaneo, M. B., Baldetti, P., Belluci, G., Bruno, R., Chioncho, G., Di Lellis, A., Shelley, E. G., Ghielmetti, A. G., Lennartsson, W., Korth, A., Rosenbauer, H., Lundin, R., Olsen, S., Parks, G. K., McCarthy, M., and Balsiger, H.: The Cluster Ion Spectrometry (CIS) Experiment, Space Science Reviews, 79, 303-350, 1997.
Rodriguez, P.: Magnetosheath electrostatic turbulence, J. Geophys. Res., 84, 917-930, 1979.

Rodriguez, P.: Magnetosheath whistler turbulence, J. Geophys. Res., 90, 6337-6342, 1985.

Rönnmark, K.: WHAMP - waves in homogeneous, anisotropic, multicomponent plasmas, Kiruna Geophysical Institute, Rep. 179, Swedish Institute of Space Phys., 1-56, 1982.

Sahraoui, F., Belmont, G., Pinçon, J.-L, Rezeau, L., Robert, P., and Cornilleau-Wehrlin, N.: Magnetic turbulent spectra in the magnetosheath: new insights, Ann. Geophys., 22, 2283-2288, 2004, http://www.ann-geophys.net/22/2283/2004/.

Sahraoui, F., Belmont, G., Rezeau, L., Cornilleau-Wehrlin, N., Pinçon, J. L., and Balogh, A.: Anisotropic turbulent spectra in the terrestrial magnetosheath as seen by the Cluster spacecraft, Phys. Rev. Letters, 96, 075002, doi:10.1103/PhysRevLett96.075002, 2006.

Schäfer, S., Glassmeier, K.-H., Narita, Y., Fornaçon, K. H., Dandouras, I., and Fränz, M.: Statistical phase propagation and dispersion analysis of low frequency waves in the magnetosheath, Ann. Geophys., 23, 3339-3349, 2005, http://www.ann-geophys.net/23/3339/2005/.

Stasiewicz, K.: Nonlinear Alfvén, magnetosonic, sound, and electron inertial waves in fluid formalism, J. Geophys. Res., 110, A03220, doi:10.1029/2004JA010852, 2005. 